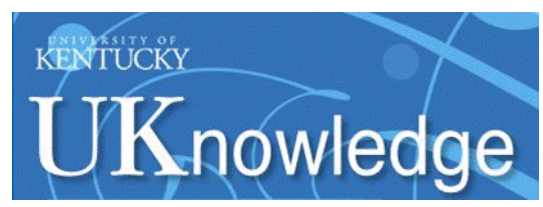

University of Kentucky

UKnowledge

$7-2015$

\title{
Observing Strategy for the SDSS-IV/MaNGA IFU Galaxy Survey
}

\author{
David R. Law \\ Space Telescope Science Institute \\ Renbin Yan \\ University of Kentucky, yanrenbin@uky.edu \\ Matthew A. Bershady \\ University of Wisconsin - Madison \\ Kevin Bundy \\ University of Tokyo, Japan \\ Brian Cherinka \\ University of Toronto, Canada
}

See next page for additional authors

Follow this and additional works at: https://uknowledge.uky.edu/physastron_facpub

Part of the Astrophysics and Astronomy Commons, and the Physics Commons

Right click to open a feedback form in a new tab to let us know how this document benefits you.

\section{Repository Citation}

Law, David R.; Yan, Renbin; Bershady, Matthew A.; Bundy, Kevin; Cherinka, Brian; Drory, Niv; MacDonald, Nicholas; Sánchez-Gallego, José R.; Wake, David R.; Weijmans, Anne-Marie; Blanton, Michael R.; Klaene, Mark A.; Moran, Sean M.; Sanchez, Sebastian F.; and Zhang, Kai, "Observing Strategy for the SDSS-IV/ MaNGA IFU Galaxy Survey" (2015). Physics and Astronomy Faculty Publications. 328.

https://uknowledge.uky.edu/physastron_facpub/328

This Article is brought to you for free and open access by the Physics and Astronomy at UKnowledge. It has been accepted for inclusion in Physics and Astronomy Faculty Publications by an authorized administrator of UKnowledge. For more information, please contact UKnowledge@lsv.uky.edu. 


\section{Observing Strategy for the SDSS-IV/MaNGA IFU Galaxy Survey}

\section{Digital Object Identifier (DOI)}

https://doi.org/10.1088/0004-6256/150/1/19

\section{Notes/Citation Information}

Published in The Astronomical Journal, v. 150, no. 1, 19, p. 1-17.

(C) 2015. The American Astronomical Society. All rights reserved.

The copyright holder has granted the permission for posting the article here.

\section{Authors}

David R. Law, Renbin Yan, Matthew A. Bershady, Kevin Bundy, Brian Cherinka, Niv Drory, Nicholas MacDonald, José R. Sánchez-Gallego, David R. Wake, Anne-Marie Weijmans, Michael R. Blanton, Mark A. Klaene, Sean M. Moran, Sebastian F. Sanchez, and Kai Zhang 


\title{
OBSERVING STRATEGY FOR THE SDSS-IV/MaNGA IFU GALAXY SURVEY
}

\author{
David R. Law ${ }^{1}$, Renbin Yan ${ }^{2}$, Matthew A. Bershady ${ }^{3}$, Kevin Bundy $^{4}$, Brian Cherinka ${ }^{5}$, Niv Drory ${ }^{6}$, \\ Nicholas MacDonald ${ }^{7}$, José R. Sánchez-Gallego ${ }^{2}$, David A. Wake $^{3,8}$, AnNe-Marie Weijmans ${ }^{9}$, Michael R. Blanton ${ }^{10}$, \\ Mark A. Klaene ${ }^{11}$, Sean M. Moran ${ }^{12}$, Sebastian F. Sanchez ${ }^{13}$, and Kai Zhang ${ }^{2}$ \\ ${ }^{1}$ Space Telescope Science Institute, 3700 San Martin Drive, Baltimore, MD 21218, USA; dlaw@ stsci.edu \\ ${ }^{2}$ Department of Physics and Astronomy, University of Kentucky, 505 Rose Street, Lexington, KY 40506-0055, USA \\ ${ }^{3}$ Department of Astronomy, University of Wisconsin-Madison, 475 N. Charter Street, Madison, WI 53706, USA \\ ${ }^{4}$ Kavli Institute for the Physics and Mathematics of the Universe, Todai Institutes for Advanced Study, \\ The University of Tokyo, Kashiwa, 277-8583 (Kavli IPMU, WPI), Japan \\ ${ }^{5}$ Dunlap Institute for Astronomy and Astrophysics, University of Toronto, $50 \mathrm{St}$. George Street, Toronto, Ontario M5S 3H4, Canada \\ ${ }^{6}$ McDonald Observatory, Department of Astronomy, University of Texas at Austin, 1 University Station, Austin, TX 78712-0259, USA \\ ${ }^{7}$ Department of Astronomy, Box 351580, University of Washington, Seattle, WA 98195, USA \\ ${ }^{8}$ Department of Physical Sciences, The Open University, Milton Keynes, MK7 6AA, UK \\ ${ }^{9}$ School of Physics and Astronomy, University of St Andrews, North Haugh, St Andrews KY16 9SS, UK \\ ${ }^{10}$ Center for Cosmology and Particle Physics, Department of Physics, New York University, 4 Washington Place, NY 10003, New York \\ ${ }^{11}$ Apache Point Observatory, P.O. Box 59, Sunspot, NM 88349, USA \\ ${ }^{12}$ Smithsonian Astrophysical Observatory, 60 Garden Street, Cambridge, MA 02138, USA \\ ${ }^{13}$ Instituto de Astronomia, Universidad Nacional Autonoma de Mexico, A.P. 70-264, 04510 Mexico D.F., Mexico \\ Received 2015 January 30; accepted 2015 May 15; published 2015 June 24
}

\begin{abstract}
Mapping Nearby Galaxies at Apache Point Observatory (MaNGA) is an integral-field spectroscopic survey that is one of three core programs in the fourth-generation Sloan Digital Sky Survey (SDSS-IV). MaNGA's 17 pluggable optical fiber-bundle integral field units (IFUs) will observe a sample of 10,000 nearby galaxies distributed throughout the SDSS imaging footprint (focusing particularly on the North Galactic Cap). In each pointing these IFUs are deployed across a $3^{\circ}$ field; they yield spectral coverage $3600-10300 \AA$ at a typical resolution $R \sim 2000$, and sample the sky with $2^{\prime \prime}$ diameter fiber apertures with a total bundle fill factor of 56\%. Observing over such a large field and range of wavelengths is particularly challenging for obtaining uniform and integral spatial coverage and resolution at all wavelengths and across each entire fiber array. Data quality is affected by the IFU construction technique, chromatic and field differential refraction, the adopted dithering strategy, and many other effects. We use numerical simulations to constrain the hardware design and observing strategy for the survey with the aim of ensuring consistent data quality that meets the survey science requirements while permitting maximum observational flexibility. We find that MaNGA science goals are best achieved with IFUs composed of a regular hexagonal grid of optical fibers with rms displacement of $5 \mu \mathrm{m}$ or less from their nominal packing position; this goal is met by the MaNGA hardware, which achieves $3 \mu \mathrm{m} \mathrm{rms} \mathrm{fiber} \mathrm{placement.} \mathrm{We} \mathrm{further} \mathrm{show} \mathrm{that} \mathrm{MaNGA}$ observations are best obtained in sets of three 15 minute exposures dithered along the vertices of a 1.44 arcsec equilateral triangle; these sets form the minimum observational unit, and are repeated as needed to achieve a combined signal-to-noise ratio of $5 \AA^{-1}$ per fiber in the $r$-band continuum at a surface brightness of $23 \mathrm{AB} \operatorname{arcsec}^{-2}$. In order to ensure uniform coverage and delivered image quality, we require that the exposures in a given set be obtained within a 60 minute interval of each other in hour angle, and that all exposures be obtained at airmass $\lesssim 1.2$ (i.e., within 1-3 hr of transit depending on the declination of a given field).
\end{abstract}

Key words: atmospheric effects - methods: observational - surveys - techniques: imaging spectroscopy

\section{INTRODUCTION}

Integral field spectroscopy (IFS) at optical and infrared wavelengths is among the most significant developments in modern observations of galaxies at all redshifts because it combines the benefits of two-dimensional photometric analysis with physical diagnostics of baryon composition and kinematics (e.g., Emsellem et al. 2004; Law et al. 2009; Bershady et al. 2010; Sánchez et al. 2012; Fabricius et al. 2014; Weijmans et al. 2014). Recent advances now enable multiobject IFS with instruments such as SAMI (Croom et al. 2012), KMOS (Sharples et al. 2013), and MaNGA (Mapping Nearby Galaxies at Apache Point Observatory; Drory et al. 2015). As a part of the 4th generation of the Sloan Digital Sky Survey (SDSS-IV), the MaNGA project (Bundy et al. 2015) bundles fibers from the Baryon Oscillation Spectroscopic Survey (BOSS) spectrograph (Smee et al. 2013) into integral-field units (IFUs) to obtain spatially resolved optical spectroscopy of 10,000 nearby galaxies over a 6 year survey. Early results obtained with prototype MaNGA hardware (Belfiore et al. 2015; Li et al. 2015; Wilkinson et al. 2015) demonstrate the richness of the data for exploring the stellar and gas composition.

Because current large-format detectors lack energy resolution throughout most of the electromagnetic spectrum, IFS has adopted a range of technical approaches to down-selecting and formatting a subset of the three-dimensional data cube of wavelength and spatial position onto a two-dimensional detector array. These approaches yield different, science-driven trades in the data-cube sampling. Simultaneous and integral coverage of the spatial field is desirable and achieved by a number of instruments using lenslets (e.g., SAURON, OSIRIS; Bacon et al. 2001; Larkin et al. 2003) or image slicers (e.g., SINFONI, MUSE; Eisenhauer et al. 2003; Bacon et al. 2010). However, the two current wide-field, multi-object, IFS 
instruments-SAMI and MaNGA-use bare-fiber arrays to minimize cost while maximizing flexibility and patrol area, but at the penalty of not achieving truly integral spatial coverage at any one time. This shortfall can be overcome by careful attention to the interplay of the hardware design of the fiber bundles and the observing strategy.

The most immediate challenge is that the MaNGA fiber bundle, composed of circular apertures with large interstitial gaps that significantly undersample the point-spread function (PSF) at the focal plane of the telescope, has a non-uniform response across each IFU. This means that (under most techniques for the reconstruction of images from the data) the appearance of objects that are small with respect to the fiber size (e.g., active galactic nuclei or $\mathrm{H}$ II regions) can vary across an IFU. The reconstructed image of such unresolved objects can either look small and circular (if the object was centered on a single fiber), large and circular (if the object was centered in the interstitial gap between three fibers), highly elongated (if the object was centered midway between two fibers), along with any range of shapes in between.

This is highly undesirable from a science standpoint, and therefore typical fiber-bundle IFU surveys (e.g., Croom et al. 2012; Sánchez et al. 2012) dither their observations. Small dithers of a fraction of the fiber spacing sample the missing points in the image plane and allow reconstructed images based on multiple, dithered exposures to achieve fairly uniform and integral spatial coverage.

This dithering is complicated by atmospheric refraction however, especially given the extremely wide spatial and spectral coverage of MaNGA. Chromatic differential refraction over the MaNGA wavelength range $(\lambda \lambda 3600-10300 \AA)$ can be comparable to the diameter of individual fibers, and field differential refraction (from variation in the amount and direction of refraction over the $3^{\circ}$ field of an SDSS plugplate) contributes similarly. These effects combine to degrade the effectiveness of a regular dithering scheme in sampling the image plane.

This paper presents simulations that explore the impact of these effects on the expected MaNGA data quality, and thereby constrain the hardware design and observing strategy for the survey. In Section 2 we give an overview of the SDSS $2.5 \mathrm{~m}$ telescope and plugplate system, along with a brief description of the MaNGA legacy hardware and IFU ferrule designs considered for the survey. We describe the basic design considerations for the survey in Section 3. Using the science requirements summarized in Section 3.1, typical integration times set by the read noise characteristics of our detectors (Section 3.2), and numerical simulations (Section 3.3) we motivate the need for dithered observations and regular hexagonal packing of the IFU fiber bundles, culminating in a baseline hardware design and observing strategy described in Section 3.4. This baseline observing strategy is significantly complicated by atmospheric differential refraction, and we discuss the impact of chromatic and field differential refraction on our data quality in Sections 4.1 and 4.2 respectively, defining a uniformity statistic $\Omega$ to describe the data quality in Section 5. Using the $\Omega$ statistic we formulate our final observing strategy in terms of visibility windows in Section 6, noting a few additional practical considerations (e.g., dithering accuracy and IFU bundle rotation) in Section 7. We summarize our conclusions in Section 8.

\section{OBSERVATORY AND HARDWARE OVERVIEW}

\subsection{Observatory and Legacy Hardware}

MaNGA operates on the SDSS $2.5 \mathrm{~m}$ telescope (Gunn et al. 2006) located at Apache Point Observatory (APO; latitude $\left.\phi=+32^{\circ} 46^{\prime} 49^{\prime \prime}\right)$. The telescope is a modified RitcheyChretien with alt-az mount that is designed with an interchangeable cartridge system that can be installed at the Cassegrain focus. The MaNGA hardware is described in greater detail by Drory et al. (2015); here we briefly review the major salient features of the system.

MaNGA has 6 cartridges, each of which contains a plugplate with a field of view $\sim 3^{\circ}$ in diameter that has been pre-drilled with holes corresponding to the locations of target galaxies into which optical fibers and IFUs can be plugged each day in preparation for a night of observing. These plates are fixed at a zero degree position angle (i.e., the on-sky orientation of the telescope focal plane coordinate reference frame is fixed).

Each MaNGA cartridge has a total of 1423 fibers (709 on spectrograph 1, 714 on spectrograph 2), corresponding to 17 science IFUs ranging in size from 19 to 127 fibers (12.5-32.5 arcsec diameter; 1247 fibers total), twelve 7-fiber mini-bundles used for spectrophotometic calibration (84 fibers total; see R. Yan et al. 2015, in preparation), and 92 single fibers used for sky subtraction that can be deployed within a $14^{\prime}$ radius of their associated IFU harness. ${ }^{14}$ Each IFU has its rotation fixed using alignment pins in the ferrules that plug into corresponding alignment holes located a short distance west of each target galaxy.

These optical fibers feed the twin BOSS (Dawson et al. 2013) spectrographs (Smee et al. 2013). The collimated beams in each spectrograph are split with a dichroic and feed a blue $(\lambda \lambda 3600-6000 \AA)$ and red camera $(\lambda \lambda 6000-10300 \AA)$. The blue cameras use blue-sensitive $4 \mathrm{k} \times 4 \mathrm{k}$ e2V CCDs while the red cameras use $4 \mathrm{k} \times 4 \mathrm{k}$ fully depleted LBNL CCDs; all cameras have $15 \mu \mathrm{m}$ pixels. Spectral resolution varies with wavelength from $R=\lambda \delta \lambda \sim 1400$ at $3600 \AA$ to $R \sim 2000$ at $6000 \AA$ (blue channel), and $R \sim 1800$ at $6000 \AA$ to $R \sim 2200$ at $10300 \AA$ (red channel; see Figure 36 of Smee et al. 2013). Spectra from each of these four cameras are extracted and processed through sky subtraction, spectrophotometric calibration, astrometric registration, and reconstructed into three-dimensional data cubes using a software pipeline (D. R. Law et al. 2015, in preparation) descended from that previously used for BOSS (idlspec2d; see Bolton et al. 2012; D. J. Schlegel et al. 2015, in preparation).

The telescope guider system is optimized for a wavelength of $\sim 5500 \AA$ and uses endoscopic fibers inserted into 16 holes in each plugplate corresponding to the locations of bright guide stars. These endoscopic fibers produce images of the guide stars on a guider camera, and the guider actively adjusts the focus, scale, rotation, and offset of the telescope focal plane to track these stars through varying weather conditions and observing angles.

\subsection{IFU Ferrule Design}

The ability of an IFU fiber bundle to deliver good, repeatable, and uniform image quality depends most

\footnotetext{
14 The physical size of the hardware components also defines a minimumdistance exclusion zone around each plugged object. These exclusion distances are $116^{\prime \prime}(7 \mathrm{~mm}), 89^{\prime \prime}(5.35 \mathrm{~mm})$, and $62^{\prime \prime}(3.7 \mathrm{~mm})$ for IFU-IFU, IFU-sky, and sky-sky fiber placement respectively.
} 


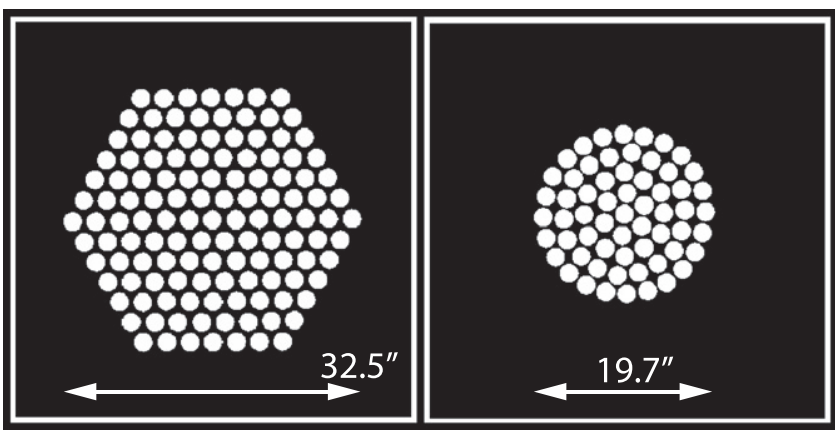

Figure 1. Fiber bundle designs considered for MaNGA (white regions represent live fiber cores). The left-hand panel shows a 127 fiber bundle for which the fibers are arranged in a regular hexagonal array (i.e., the final MaNGA IFU design; shown here is as-built harness ma024); the right-hand panel shows an example bundle of 61 fibers in a circular packing arrangement based on that adopted by the SAMI team for use at the Australian Astronomical Observatory (Croom et al. 2012, compare their Figure 3). Although the hexagonal arrangement of fibers has greater regularity, the circular arrangement has greater effective filling factor since the protective buffers are stripped.

fundamentally on the arrangement of fibers within the bundle; while dithering (Section 3.3.2), differential refraction (Section 4), and other considerations are important, the fiber placement sets the basis for the sampling regularity of the entire survey.

As described by Drory et al. (2015), the MaNGA fibers have an inner light-sensitive core diameter (ID) of $120 \mu \mathrm{m}$ (corresponding to 2.0 arcsec in the telescope focal plane) and an outer diameter $(\mathrm{OD})$ of $151.0 \pm 0.5 \mu \mathrm{m}$ with their protective buffers and cladding. We originally considered two kinds of fiber bundles for MaNGA, as illustrated in Figure 1. The first was a circular bundle of fibers that maximizes the filling factor of light-sensitive fiber cores relative to the total IFU footprint by chemically stripping the protective buffers from the ends of each fiber. As developed for the SAMI survey by BlandHawthorn et al. (2010), these "Sydney-style" bundles maximize the effective filling factor at the cost of decreased fiber throughput due to focal ratio degradation (FRD), greater fragility of the glass cores, and irregular fiber packing due to the circular ferrule geometry. Based on the numerical performance simulations described in Section 3.3.3, we prototyped (and ultimately chose to adopt) a second style of fiber bundle composed of a regular arrangement of buffered fibers within a tapered hexagonal ferrule for which we pioneered a novel construction technique (see details in Drory et al. 2015). While reaching lower effective filling factor, this technique improves fiber throughput, ${ }^{15}$ decreases breakage, ${ }^{16}$ and (by virtue of its hexagonal geometry) permits extremely regular fiber placement within each IFU.

\footnotetext{
15 A conservative estimate can be made by comparing Figure 4 of Croom et al. (2012) to Figure 11 of Drory et al. (2015): MaNGA achieves $95 \% \pm 1 \%$ throughput with an exit f-ratio of $f / 4$ for fibers fed at $f / 5$. In contrast, the original SAMI bundles achieved $50 \%-75 \%$ throughput with an exit f-ratio of $\mathrm{f} /$ $3.15 \mathrm{fed}$ at $\mathrm{f} / 3.4$. We note that the FRD of even the second-generation SAMI bundles (Figure 5 of Bryant et al. 2014) is sufficiently large that it would require our optics to be $40 \%$ larger in area to collect the same ensquared energy given the Sloan telescope feed.

${ }^{16}$ After $\sim 6$ months of operation, 7 individual fibers within IFUs have broken ( 1 in manufacturing, 1 in assembly, 5 in operation), representing $<0.1 \%$ of the total. Detailed statistics on the breakage frequency of stripped, fused fiber bundles are unknown but would have represented a significant cost increase in manufacturing.
}

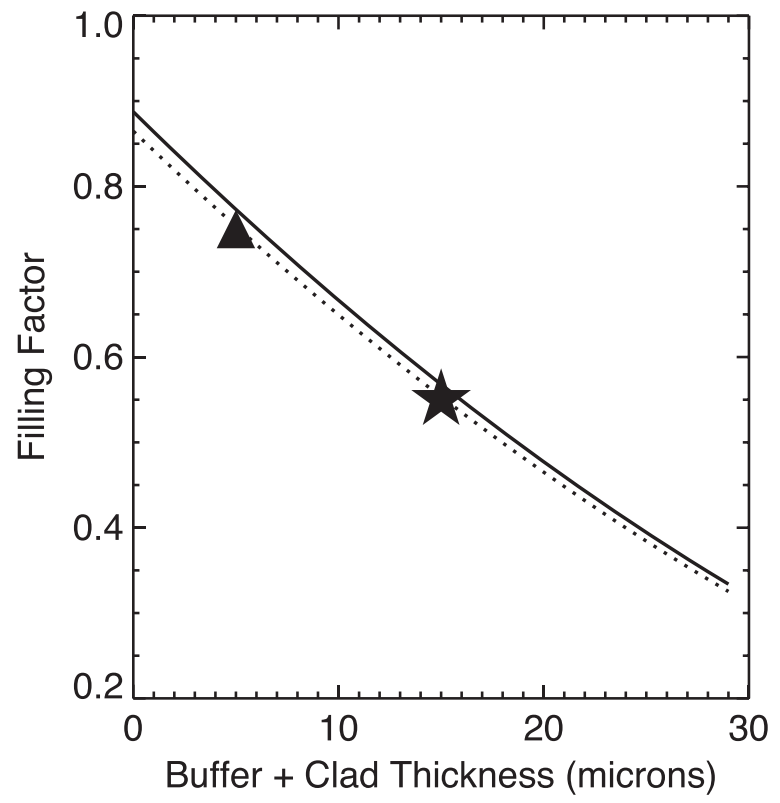

Figure 2. Effective IFU filling factor (live fiber core area divided by total IFU footprint) as a function of buffer thickness for an ideal 127 fiber (solid line) and a 19 fiber (dotted line) hexagonal IFU. The small difference between the solid and dotted lines represents the diminishing importance of edge effects in the hexagonal footprint as the IFU area increases. The filled star represents the measured 56\% filling factor of the as-built 127 fiber MaNGA IFUs (Drory et al. 2015), which is consistent with theoretical expectations. The filled triangle shows the $75 \%$ filling factor of the SAMI survey bundles $(5 \mu \mathrm{m}$ cladding) for comparison.

The theoretical effective fiber packing density of the hexagonal IFUs can be defined as the ratio of the total fiber core area $\left(A_{\text {core }}\right)$ to the area of the hexagon circumscribing the fiber bundle $\left(A_{\text {hex }}\right)$, where:

$$
\begin{gathered}
A_{\text {hex }}=\frac{\sqrt{3}}{2} d^{2}\left(\sqrt{3} N_{\mathrm{R}}+1\right)^{2} \\
A_{\text {core }}=\pi\left(\frac{d-2 t}{2}\right)^{2}\left(1+3 N_{\mathrm{R}}\left(N_{\mathrm{R}}+1\right)\right) .
\end{gathered}
$$

Here $d=151 \mu \mathrm{m}$ is the OD of an individual fiber, $t=15.5 \mu \mathrm{m}$ is the thickness of the fiber buffer and cladding, and $N_{\mathrm{R}}$ is the number of "rings" in the bundle $\left(N_{\mathrm{R}}=2\right.$ for a 19 fiber IFU, and $N_{\mathrm{R}}=6$ for a 127 fiber IFU). In Figure 2 we plot the effective filling factor $f=A_{\text {core }} / A_{\text {hex }}$ as a function of the buffer thickness $t$. In accord with these predictions, the prototype circular Sydney-style bundles (whose fibers are chemically etched to an OD of $\sim 132 \mu \mathrm{m}$ ) achieve a filling factor of $\sim 70 \%$, while the as-built hexagonal bundles with fully buffered fibers achieve a filling factor of $56 \%$.

\section{BASIC CONSIDERATIONS}

\subsection{Required Performance}

Since the fiber bundles consist of $2^{\prime \prime}$ diameter circular apertures separated by large interstitial gaps, each exposure will significantly undersample the PSF at the focal plane of the telescope (typically $\sim 1^{\prime \prime} .5$ ) and produce a non-uniform response function across the face of each IFU. We require that the MaNGA IFUs deliver sufficiently uniform performance that physical structures do not vary in shape as a function of where they happen to fall within the IFU (i.e., a circular star 


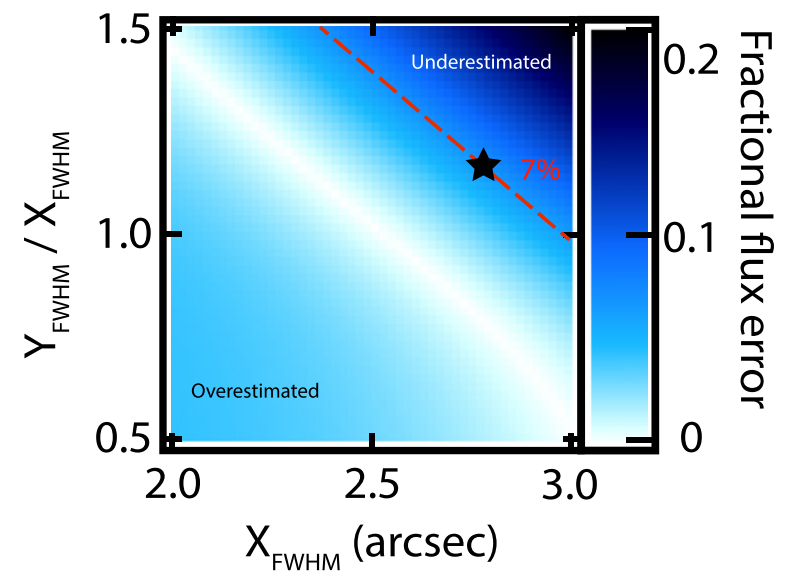

Figure 3. Fractional error in the recovered flux from a point source if the assumed FWHM and axis ratio were incorrect. The reference source is taken to have a circular gaussian PSF with FWHM 2.5 arcsec; integrating the flux within a $2.5 \mathrm{rms}$ width aperture nominally encloses $95.7 \%$ of the total flux. If the actual FWHM is smaller (larger) than the model along any dimension the total flux enclosed by the aperture increases (decreases), resulting in an overestimate (underestimate) of the total flux. The dashed red line indicates the $7 \%$ error threshhold set by the MaNGA SRD; the solid black star indicates our adopted limits on the allowable variability of the delivered MaNGA PSF (15\% in axis ratio, and $15 \%$ in circularly averaged PSF FWHM).

forming region within a galaxy should appear circular in the final MaNGA data cube regardless of whether it is in the center or the outskirts of the galaxy).

A convenient way to place a limit on the level of uniformity required is to ensure that variations in the $2 \mathrm{~d}$ PSF of the reconstructed MaNGA data cubes do not significantly impact measurements of the Balmer decrement or BPT-style (e.g., Baldwin et al. 1981) line ratio diagrams. Since atmospheric differential refraction shifts the effective position of each fiber as a function of wavelength (Section 4), [O II] and $\mathrm{H} \alpha$ observations of a given $\mathrm{H}$ in region for instance will be obtained with a slightly different configuration of fiberswhile $\mathrm{H} \alpha$ emission may be centered in a given fiber, spatially coincident $\left[\mathrm{O}_{\mathrm{II}}\right]$ emission may be centered in the interstitial region between fibers.

As outlined in the MaNGA Science Requirements Document (SRD; see R. Yan et al. 2015, in preparation), relative spectophotometry between $\left[\mathrm{O}_{\mathrm{II}}\right](\lambda=3727 \AA)$ and $\mathrm{H} \alpha$ $(\lambda=6564 \AA)$ must be accurate to $7 \%$ or better in order to obtain the desired constraints on the star formation rate (SFR) and nebular metallicity within galaxies. We therefore explore how this required spectrophotometric accuracy translates to limits on the spatial variability of the MaNGA PSF.

We begin by assuming that the PSF in a typical MaNGA reconstructed data cube can be characterized by a circular gaussian with a FWHM of 2.5 arcsec (as we discuss at greater length in D. R. Law et al. 2015, in preparation, this model is a good approximation to the MaNGA commissioning data). Using typical aperture photometry techniques, a circular aperture of radius $2.66 \operatorname{arcsec}$ (i.e., 2.5 times the radial scalelength of the PSF) would nominally enclose $96 \%$ of the total flux. ${ }^{17}$ In Figure 3 we illustrate how deviations from the nominal PSF model would affect this total; as the PSF becomes

\footnotetext{
17 If we were to adopt a PSF model with more power in the wings, or shrink the size of the circular aperture the variability between different PSF shapes would increase and lead to more stringent constraints on the allowable variability in the delivery MaNGA PSF.
}

broader or more elongated the flux contained within the fixed aperture decreases, meaning that the derived aperture-corrected total fluxes would be in error. ${ }^{18}$ In particular, we find that an error of $20 \%$ in the profile FWHM and $15 \%$ in the profile minor/major axis ratio is sufficient to bias the resulting flux measurements at the $7 \%$ level (filled star in Figure 3).

Similarly, in order to ensure that our limiting fluxes for undetected nebular transition features are accurate at the $7 \%$ level we also require that the signal-to-noise ratio $(\mathrm{S} / \mathrm{N})$ of our data is constant at the $7 \%$ level across each IFU. Since the limiting flux is proportional to the square root of the exposure time, this translates to a requirement that the exposure time is effectively constant across each IFU at the $15 \%$ level.

These three metrics (circularity, FWHM, and S/N) therefore set our requirements on the uniformity of the reconstructed image profile such that the calibrated fluxes derived from MaNGA data cubes are accurate at the $7 \%$ level. Ideally, however, we would prefer that spatial sampling issues not dominate the flux calibration accuracy budget for the MaNGA data cubes, and we therefore set a goal of achieving photometric performance at the $3.5 \%$ level where possible. The MaNGA hardware construction, dithering pattern, and observing strategy is therefore set by the following three highlevel considerations.

1. The reconstructed FWHM of all angular resolution elements in a bundle should vary by $<10 \%$ (goal) or $20 \%$ (requirement) across each IFU.

2. The reconstructed minor-to-major axis ratio of all resolution elements in a bundle should be $b / a \geqslant 0.93$ (goal) or 0.85 (requirement) across each IFU.

3. The effective integration time of all resolution elements in a bundle should vary by $<7 \%$ (goal) or $15 \%$ (requirement) across each IFU.

\subsection{Integration Time}

The total integration time is set by our requirement that MaNGA reach a S/N of $5 \AA^{-1}$ fiber $^{-1}$ in the $r$-band continuum at a surface brightness of $23 \mathrm{AB} \mathrm{arcsec}{ }^{-2}$. As described by D. A. Wake et al. (2015, in preparation) and R. Yan et al. (2015, in preparation) the typical integration time per plate to reach this target is anticipated to be about $3 \mathrm{hr}$ in median conditions. In good conditions however the required time could be as low as 1.5-2 hr, and for particularly low-latitude fields the required time could be as much as $4-5 \mathrm{hr}$. This substantial variation in total exposure time requires an observing strategy flexible enough to accommodate it.

The optimal integration time for individual exposures is constrained by the MaNGA hardware and typical background sky spectrum at APO. One of the strengths of MaNGA is the high throughput of the BOSS spectrographs shortward of $4000 \AA$, and we therefore integrate each exposure for long enough that the shot noise from the background sky spectrum and detector dark current exceeds the read noise. The total

\footnotetext{
${ }^{18}$ If the goal were to measure the flux from a single bright source whose structure is known a priori to be effectively a point source then the actual light profile could be measured at each wavelength and the aperture adjusted accordingly. However, such a priori knowledge of the intrinsic source structure cannot generally be assumed. Similarly, we assume that wavelength-dependent variations from the $\lambda^{-1 / 5}$ Kolmogorov atmospheric turbulence profile are taken into account in determining the appropriate aperture.
} 


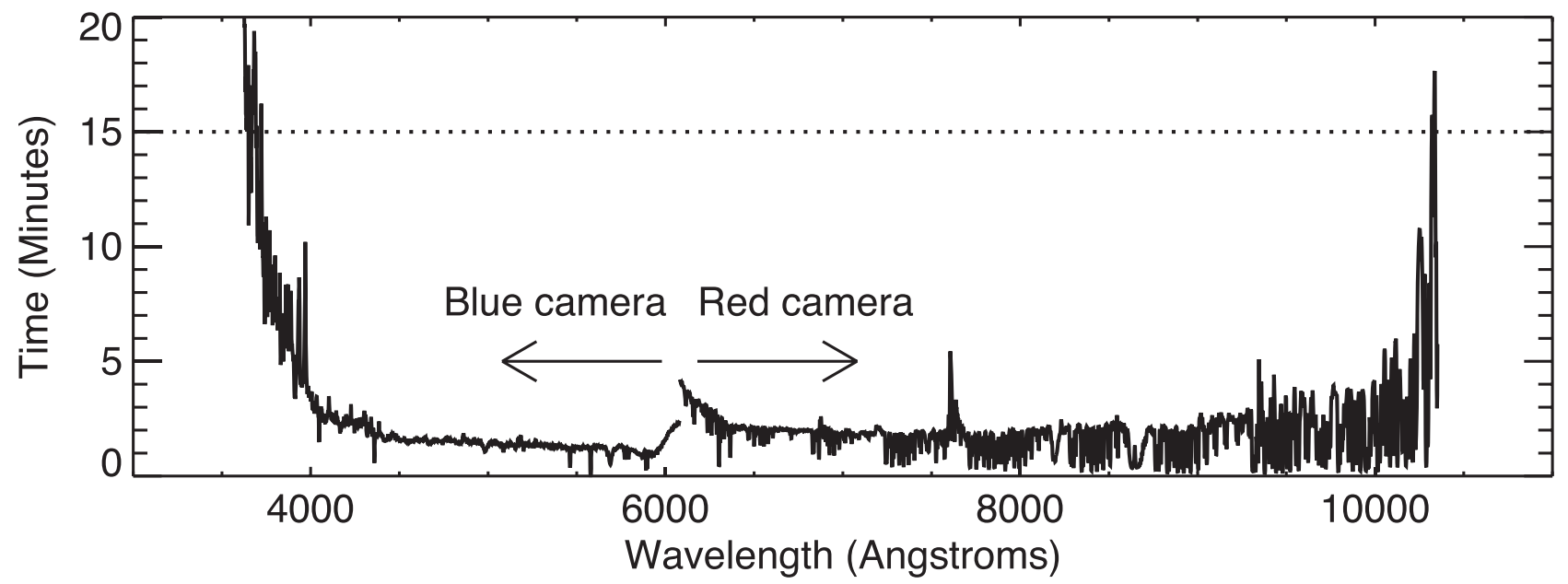

Figure 4. Exposure time $t_{\min }$ required for a typical MaNGA dark-time sky spectrum to be dominated by Poisson noise from the background sky plus detector dark current. The break around $6000 \AA$ represents the dichroic break between red and blue channels; in reality there is a $\sim 300 \AA$ overlap between these channels. Strong features longwards of $\sim 8000 \AA$ are due to bright $\mathrm{OH}$ sky lines. The dashed line indicates the adopted 15 minute exposure time.

noise $N$ as a function of wavelength is given by

$$
N(\lambda)=\sqrt{\left(f_{\mathrm{s}}(\lambda)+f_{\mathrm{d}} n_{1}\right) t+n_{1} N_{\mathrm{r}}^{2}}
$$

where $f_{\mathrm{s}}(\lambda)$ is the background sky spectrum in units of $\mathrm{e}^{-}$ minute $^{-1}$ per spectral pixel, $f_{\mathrm{d}}$ is the dark current in $\mathrm{e}^{-}$pixel $^{-1}$ minute ${ }^{-1}, N_{\mathrm{r}}$ is the read noise in $\mathrm{e}^{-}$pixel $^{-1}, n_{1}=3$ pixels is the spatial width of a spectrum on the detector (see discussion by D. R. Law et al. 2015, in preparation), and $t$ is the integration time of an exposure in minutes. Rearranging Equation (3) we find the time $t_{\min }$ required for the combined sky background and dark current to equal the read noise:

$$
t_{\min }(\lambda)=\frac{n_{1} N_{\mathrm{r}}^{2}}{f_{\mathrm{s}}(\lambda)+f_{\mathrm{d}} n_{1}}
$$

We estimate $f_{\mathrm{s}}(\lambda)$ for a typical MaNGA dark-time observation using commissioning data from all-sky plate 7341 (i.e., a calibration plate for which all IFUs target regions of blank sky) observed on MJD 56693. ${ }^{19}$ Following the data model outlined by D. R. Law et al. (2015, in preparation), we take the FLUX array of the reduced $\mathrm{mgFrame}$ file (in units of flatfielded $\mathrm{e}^{-}$per spectral pixel), multiply by the SUPERFLAT array to obtain spectra in raw $\mathrm{e}^{-}$per spectral pixel, and combine $\sim 600$ individual fiber spectra to construct an extremely highprecision model of the background sky. We take the detector read noise to be $R_{\mathrm{n}}=2.0(2.8) \mathrm{e}^{-}$pixel $^{-1}$, and the dark current to be $0.033(0.066) \mathrm{e}^{-}$pixel $^{-1}$ minute ${ }^{-1}$ for the blue (red) camera (see Table 4 of Smee et al. 2013). ${ }^{20}$

We plot $t_{\min }$ as a function of wavelength in Figure 4, and note that the sky background rapidly dominates over read noise at almost all wavelengths, especially in the vicinity of strong $\mathrm{OH}$ atmospheric emission lines in the near-IR. The upturn in $t_{\text {min }}$ shortward of $4000 \AA$ represents the falloff in blue sensitivity of the detectors, but an integration time of 15 minutes per exposure ensures that observations are shotnoise dominated for all $\lambda>3700 \AA$.

Although an integration time of longer than 15 minutes would further decrease the contribution of read noise to the

\footnotetext{
19 MJD (Modified Julian Date) 56693 corresponds to 2014 February 5.

20 The dark current is typically $\lesssim 2 \%$ of the dark-time sky background signal.
}

total error budget, such longer integrations are undesirable because of the cosmic ray event rate recorded by the redchannel detectors. In practice, the maximum integration time of each exposure is also limited by differential atmospheric refraction considerations (see Section 7.2), and we therefore adopt a nominal time of 15 minutes per exposure. Each completed plate will therefore consist of $\sim 6-20$ exposures in order to reach the target depth.

\subsection{Numerical Simulations \\ 3.3.1. Simulation Method}

In order to assess the relative performance of different IFU bundles and observing techniques we perform a series of numerical simulations designed to test the uniformity of their response to unresolved point sources (for which spatial structure is most pronounced). Adopting a working box size of $\sim 45 \times 45$ arcsec with simulated pixels spaced every $0.1 \operatorname{arcsec}$ we first compute the footprint of a given IFU; this defines a mask image for which each fiber in the IFU is associated with a given set of pixels in the telescope focal plane that its light-sensitive core subtends. We then create an input "image" to be observed by the simulated MaNGA IFUs by convolving a delta function by a model of the PSF at the focal plane of the SDSS $2.5 \mathrm{~m}$ telescope. This focal-plane PSF is taken to be the sum of two Gaussian profiles with FWHM $\theta$ and $2 \theta$ respectively (where $\theta=1.4 \operatorname{arcsec}$ is the FWHM of the median atmospheric seeing profile divided by 1.05) and peak amplitude ratio of $9 / 1 .{ }^{21}$ This input image is convolved with the top-hat fiber mask to determine the total amount of light received by each fiber; although the present simulation considers only a single input image the technique is immediately generalizable to multi-wavelength input image slices.

We reconstruct a two-dimensional image from the individual fiber fluxes using a flux-conserving variant of Shepards method

\footnotetext{
${ }^{21}$ Mathematically, this is equivalent to the linear sum of $9 / 13$ times the input image convolved with a Gaussian of FWHM $\theta$ plus 4/13 times the input image convolved with a Gaussian of FWHM $2 \theta$. This profile provides a reasonable approximation of the on-axis SDSS focal plane PSF, matching the inner parts of the profile well and accounting for most of the flux in the outer wings (J. E. Gunn 2015, private communication).
} 


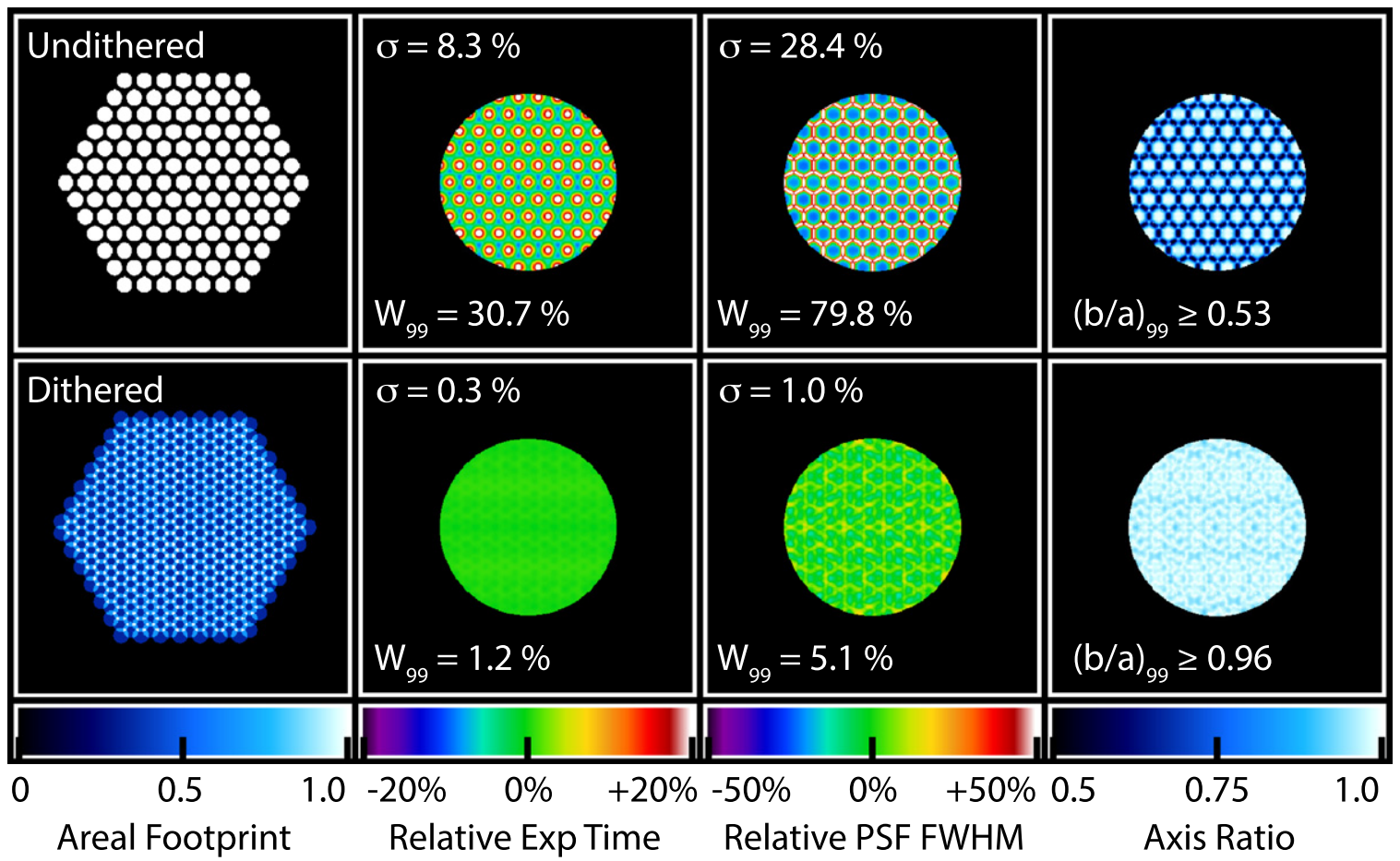

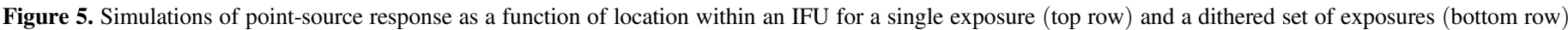

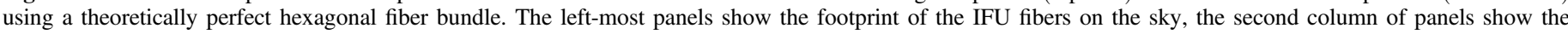

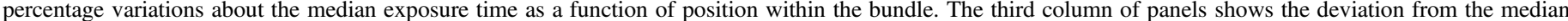

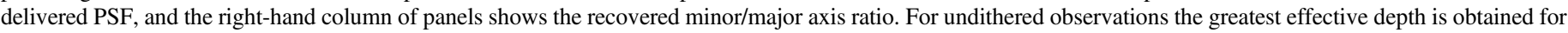

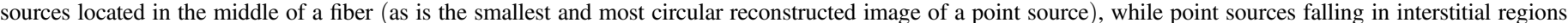

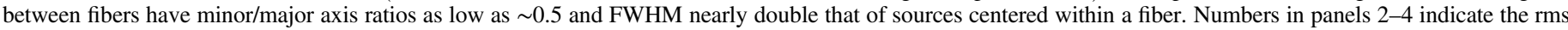
deviation between values $(\sigma)$, the $3 \sigma$ width encompassing $99 \%$ of all values $\left(W_{99}\right)$, and the minor/major axis ratio above which $99 \%$ of point lie $\left.((b / a))_{99}\right)$.

(inverse-distance weighting) similar to that used by the CALIFA survey (Sánchez et al. 2012). As part of MaNGA design simulations we explored alternative methods of image reconstruction such as drizzling (e.g., as adopted by SAMI, see Sharp et al. 2015), thin-plate-spline fits, minimum curvature surface fits, and kriging. As discussed by D. R. Law et al. (2015, in preparation) the modified Shepard's method yielded the best results, and here we adopt the same parameters (e.g., final spaxel scale of $0.5 \mathrm{arcsec}$ ) as used by the MaNGA Data Reduction Pipeline (DRP) for genuine survey data. The reconstructed image is fit with a $2 \mathrm{~d}$ Gaussian model to determine its FWHM and axial ratio; major axis rotation is left as a free parameter.

This exercise is repeated for delta functions located in each of the 0.1 arcsec grid squares that lie within the central $75 \%$ of the IFU fiber bundle footprint (i.e., ignoring edge effects from point sources located on the outer ring of an IFU), resulting in $\sim 40,000$ simulated points across a 127 fiber IFU bundle. In Figure 5 (top row) we plot the on-sky footprint of a hexagonal fiber array, along with the variations in effective exposure time (exposure time multiplied by the fraction of the total light that is collected by fibers rather than being lost to interstitial regions), FWHM, and minor-to-major axis ratio of the reconstructed PSF as a function of the location of the point source within the fiber bundle. As anticipated, we note that all three quantities vary substantially across a given IFU in a single exposure.

We quantify these results by calculating the rms of the distributions in effective exposure time and reconstructed PSF FWHM (relative to the median values as $\left[X-X_{\text {median }}\right] / X_{\text {median }}$ ), the $3 \sigma$ width $W_{99}$ encompassing $99 \%$ of these values, and the $99 \%$ lower bound for the minor-to-major axis ratio. For a single exposure, the effective integration time varies by $W_{99}=30.7 \%$ around the median $^{22}$ value; unsurprisingly, the greatest fraction of the total light is recorded for objects that are centered in a fiber, while the least amount is recorded for objects in interstitial regions. Similarly, the reconstructed PSF FWHM varies by almost $80 \%$ (from $\sim 2$ to 4 arcsec) depending on where a source falls with respect to the fiber grid, and the minor-to-major axis ratio $b / a$ of the reconstructed image varies from $\sim 0.5-1.0$ (99\% of values $b / a \geqslant 0.53$ ).

In practical terms, this means that an unresolved $\mathrm{H}$ in region observed with such an IFU for just a single exposure may appear to be compact and circularly symmetric if it lands directly in the middle of a fiber, elongated and skinny if it falls directly between two fibers, or large and triangular if it falls midway between three adjacent fibers. ${ }^{23}$ Allowing for the effects of chromatic differential refraction (Section 4.1), this means that a single such $\mathrm{H}$ in region may simultaneously be sampled by all three different such configurations at different wavelengths.

\subsubsection{Dithering}

The sampling irregularities from fiber-bundle IFUs with substantial interstitial light losses are well known from previous

\footnotetext{
${ }^{22}$ The median effective exposure time is just the filling factor $(0.56)$ times the actual exposure time.

23 Strictly, a single exposure simply does not have the spatial sampling in these cases to discriminate (for instance) between an unresolved point source and an elongated source.
} 

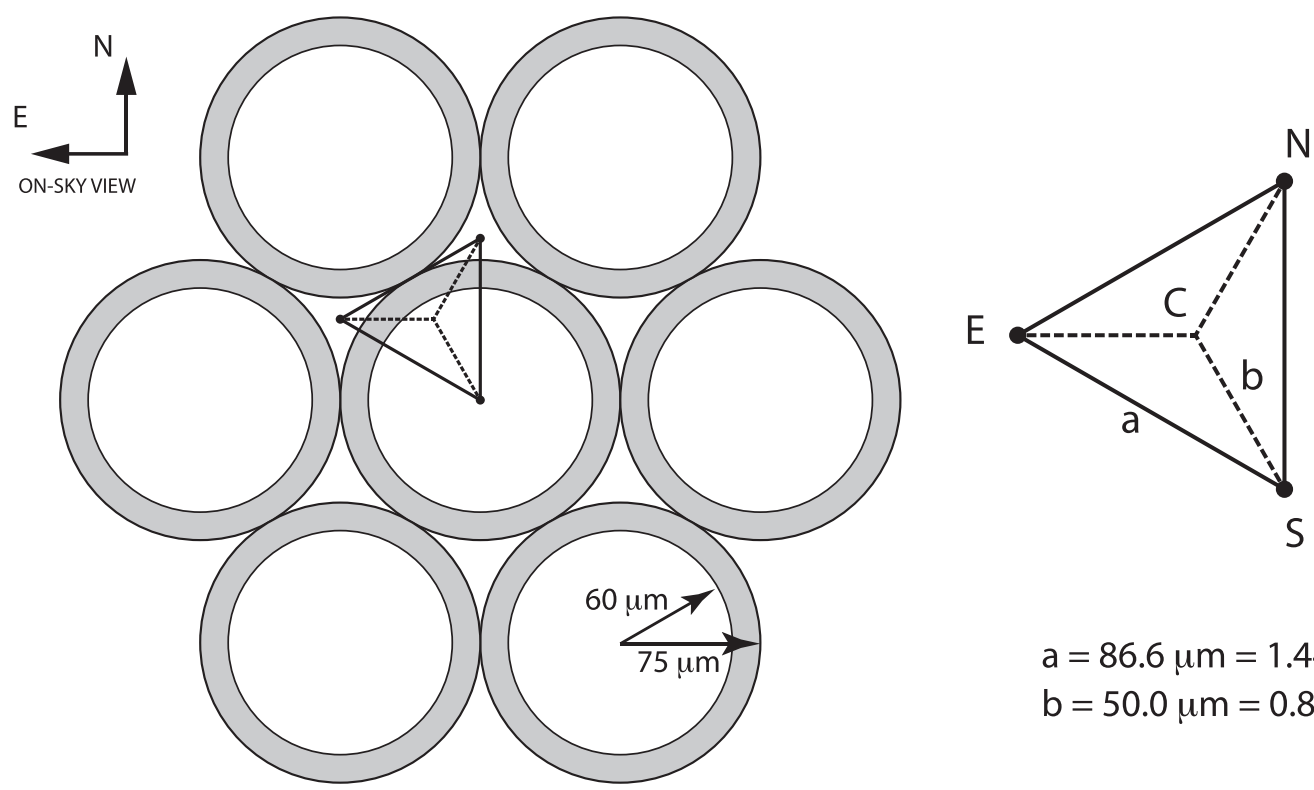

$$
\begin{aligned}
& a=86.6 \mu \mathrm{m}=1.44^{\prime \prime} \\
& b=50.0 \mu \mathrm{m}=0.83^{\prime \prime}
\end{aligned}
$$

Figure 6. Schematic diagram of the 7 central fibers within a hexagonally packed MaNGA IFU, showing the 120 micron diameter fiber core and surrounding cladding plus buffer. The triangular figure shows the relative positions of the three dither positions; the fiber bundle is located at position "S." The central (C) "home" position is labeled, along with the north $(\mathrm{N})$, south $(\mathrm{S})$, and east $(\mathrm{E})$ dither positions. The nominal plate scale of the SDSS telescope is $217.7358 \mathrm{~mm}$ degree ${ }^{-1}$, or 60.48 microns $\operatorname{arcsec}^{-1}$.

IFU surveys (e.g., Sánchez et al. 2012; Sharp et al. 2015), and can be largely overcome by obtaining dithered observations. The geometry of the hexagonal fiber arrangement readily lends itself to a fixed triangular three-point dithering scheme that effectively fills the interstitial regions as illustrated in Figure 6. Repeating the simulations performed in Section 3.3.1 with such dithered observations, we find that the combined data from just three exposures is able to achieve remarkably uniform image quality at all locations within a single IFU (Figure 5, bottom row). In contrast to the undithered case, 3 point dithering delivers effective exposure time constant to within $0.3 \% \mathrm{rms}$, FWHM of $2.69 \pm 0.01$ arcsec, and ellipticity $\leqslant 0.04$. This uniformity easily meets the MaNGA science requirements described in Section 3.1.

Logically, the 3 point dithering pattern could be expanded to a regular 9 point pattern that also provides uniform coverage of the interstitial gaps, but with a finer sampling of the image plane. Although simulations suggest that this could provide $\sim 10 \%$ improvement in the delivered PSF FWHM, such gains were not realized on-sky in tests with the MaNGA prototype hardware. This lack of improvement with respect to theoretical calculations is likely due to a confluence of numerous complicating factors, including degradation of the nominal dithering pattern by atmospheric refraction (see Section 4), variations in fiber-to-fiber sensitivity, and changes in seeing and transparency conditions between exposures (see Section 6.2).

\subsubsection{Fiber Packing Regularity}

The gains achievable with such dithering depend fundamentally on the uniformity of each IFU fiber bundle so that a single telescope offset can simultaneously dither each of our 29 IFUs (17 science and 12 calibration bundles) across the $3^{\circ}$ field such that their fibers align with the interstitial gaps from the previous exposure. If fibers are not located at regular positions within every IFU, the dithering will not be able to uniformly sample the image plane. We explore the effect of fiber packing irregularity by repeating our earlier simulations with the introduction of a random perturbation to the position of each fiber in the simulated IFU fiber bundle, such that each fiber is slightly offset from its nominal position by some distance drawn randomly from a Gaussian distribution with a given rms. Each simulated IFU bundle is observed with a nominal 3 point dither pattern as defined by Figure 6. Additionally, we simulate the effect of observing the circular Sydney-style fiber bundle with a 7 point dither pattern (based on that adopted by the SAMI survey) that compensates for the irregular fiber placement with greater filling factor and a larger number of dithered sampling points.

As indicated by Figure 7, neither the dithered Sydney-style circular fiber bundle nor the $20 \mu \mathrm{m}$ tolerance hexagonal fiber bundles meet our target regularity goals, with a recovered PSF FWHM $^{24}$ varying by $>20 \%$ over the extent of an IFU (i.e., $2.66 \pm 0.12$ arcsec with $99 \%$ values ranging from $\sim 2.3-2.9 \mathrm{arcsec}$ ), and minor/major axis ratios as low as b/a $\sim 0.8$. In contrast, using a hexagonal fiber array constructed to a tolerance of $5 \mu \mathrm{m} \mathrm{rms}$ with a 3 point dither pattern we expect to achieve a PSF FWHM that varies by less than $10 \%$ over a given IFU.

As detailed by Drory et al. (2015), the as-built MaNGA fiber bundles meet and exceed our target threshold with a typical fiber placement accuracy of $3 \mu \mathrm{m} \mathrm{rms}$. Using the as-measured fiber metrology ${ }^{25}$ for 127 fiber MaNGA bundle ma024, we simulate the anticipated performance using this fiber bundle in row $\mathrm{E}$ of Figure 7 . With a nominal dither pattern we expect to achieve a PSF FWHM which varies by less than $7 \%$ over an IFU (i.e., $2.66 \pm 0.01$ arcsec with $99 \%$ values ranging from $\sim 2.6$ to 2.7 arcsec), and has a nearly circular profile everywhere with $b / a>0.95$.

\footnotetext{
${ }^{24}$ We quote the average of the minor- and major-axis FWHM values.

25 The final placement of individual fibers within an IFU can be measured to an accuracy of better than $1 \mu \mathrm{m}$ (Drory et al. 2015).
} 


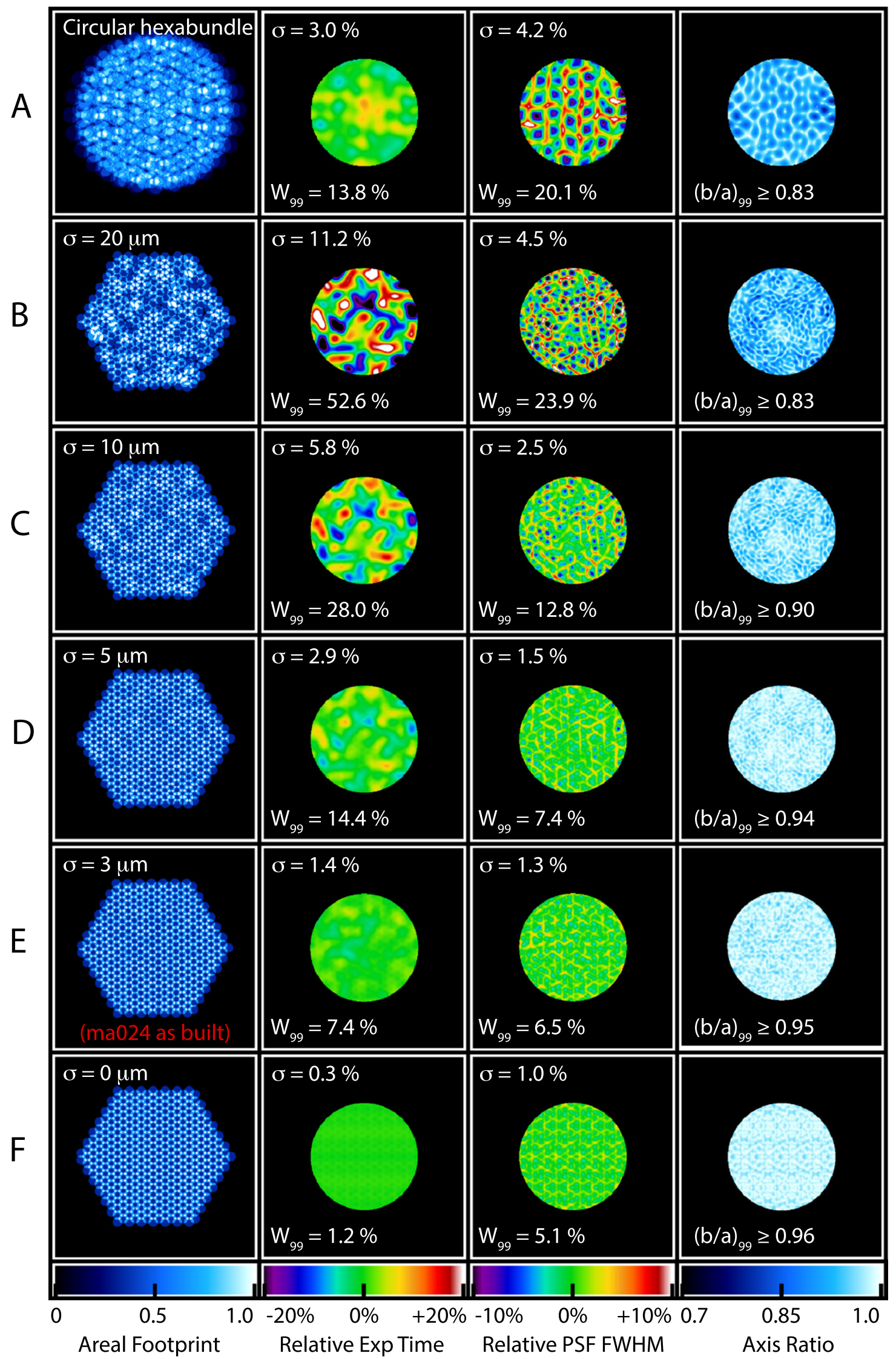

Figure 7. As Figure 5, but showing simulated point-source response as a function of location in an IFU for dithered observations of fiber bundles built to a variety of specifications. Row A simulates a Sydney-style 61 fiber bundle using a 7 point dither pattern. Rows B-F simulate a 3 point dither pattern applied to a hexagonal arrangement of 127 fibers with varying rms deviations of each fiber from the nominal position $(\sigma=0-20 \mu \mathrm{m})$. Note that for display purposes panel A is zoomed in slightly compared to panels B-F. In order to meet our uniformity criteria we require $\sigma<5 \mu \mathrm{m}$, which our as-built IFUs achieve (row E). 


\subsection{Baseline Observing Strategy}

The dithered observing simulations presented in Section 3.3.2 and exposure time requirements described in Section 3.2 motivate a nominal observing scheme in which targets are observed in sets of 3 dithered exposures (N-S-E) of 15 minutes each. Given the regularity of fiber placement with each IFU and the locator pins that constrain each IFU to have the same position angle, correctly dithered exposures can be simultaneously obtained for all IFUs on a given plate by simply offsetting the telescope pointing with respect to the guide stars. Since the coverage and image quality of a single set of three dithered exposures is known to be acceptably uniform, the total summed coverage of $N$ such sets will also be uniform and have a depth of $0.75 \mathrm{Nhr}$, allowing us to simply observe additional sets of 3 exposures until the combined data reaches our target $\mathrm{S} / \mathrm{N}$ of $5 \AA^{-1}$ fiber $^{-1}$ in the $r$-band continuum at a surface brightness of $23 \mathrm{AB} \operatorname{arcsec}^{-2}$.

Such a scheme provides us with considerable flexibility to adjust our total exposure time in 45 minute increments without adversely impacting the delivered data quality whether there are 6 or 20 total exposures for a given galaxy. It is this flexibility as much as the dithered performance simulations themselves that drives us to adopt the regular hexagonal fiber arrays for MaNGA rather than the SAMI-style circular fiber bundles, which rely upon a large number of exposures at many different dither positions to statistically fill in the interstitial gaps. ${ }^{26}$ However, since this technique relies upon tightly controlling the fiber locations to provide uniform coverage we must properly mitigate a variety of effects that will act to degrade this uniformity, and this goal in turn drives many aspects of the survey operation.

\section{ATMOSPHERIC REFRACTION}

As a photon passes through the Earth's atmosphere it is refracted by variations in the density of the air. Under the usual assumption of a plane-parallel atmosphere with a vertical density gradient this bends the light from an astronomical target along the parallactic angle (the great circle connecting the target and the observers local zenith), causing astronomical objects to appear slightly higher in the sky than they truly are. Atmospheric refraction introduces significant optical distortions that adversely affect our ability to dither our IFU observations to the desired accuracy. Loosely speaking, the effects can be split into chromatic differential refraction and field differential refraction which we detail below.

\subsection{Chromatic Differential Refraction}

Atmospheric refraction is a function of atmospheric conditions (temperature, pressure, and relative humidity), zenith distance (i.e., the amount of atmosphere that an incoming photon must traverse), and wavelength. The impact of such refraction on astronomical observations has been studied at some length in the literature (e.g., Filippenko 1982; Cuby et al. 1998, and references therein); we adopt estimates of the magnitude of refraction $r$ at a given wavelength relative to a fixed "guide" wavelength developed by Enrico Marchetti for ESO. ${ }^{27}$ The direction of the refraction is along the local altitude

\footnotetext{
${ }^{26}$ Additionally, the hexagonal tapered ferrule construction technique can be scaled up to bundles with large numbers of hexagonal "rings" without significantly degrading the packing regularity.

${ }_{27}$ See http://www.eso.org/gen-fac/pubs/astclim/lasilla/diffrefr.html.
}

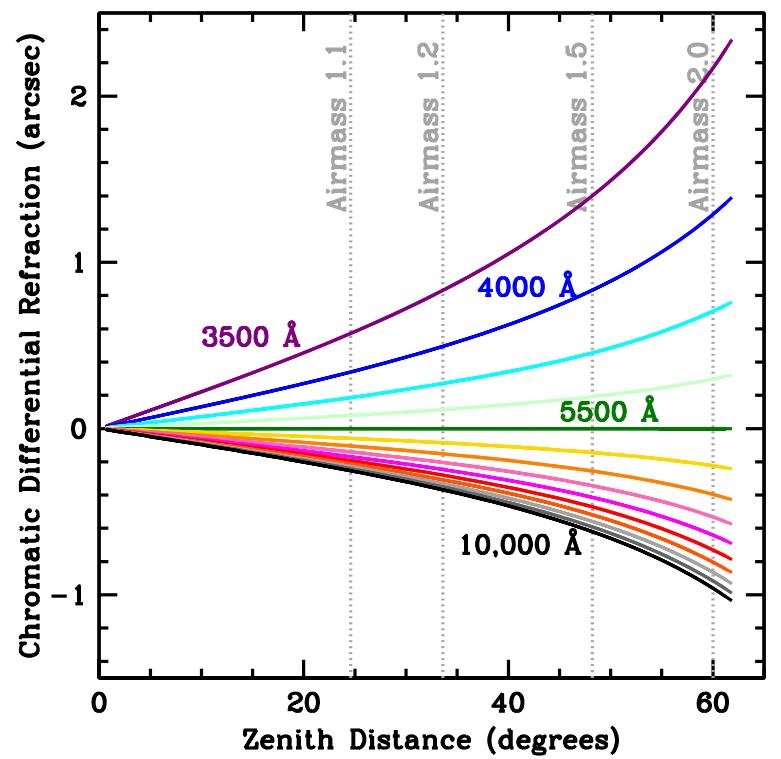

Figure 8. Differential atmospheric refraction in arcsec of altitude relative to $5500 \AA$ for the MaNGA wavelength range as a function of zenith distance. Calculations assume median conditions for APO with air temperature $10.5^{\circ} \mathrm{C}$, $24.5 \%$ relative humidity, and atmospheric pressure of 730 mbar.

vector for a given star; this corresponds to the parallactic angle $\eta$ defined by the spherical triangle with vertices at the star, the celestial pole, and the local zenith.

$$
\tan \eta=\frac{\sin h \cos \phi \cos \delta}{\sin \phi-\sin \delta \cos z}
$$

where $h$ is the hour angle ( $h>0$ toward the west), $\phi$ is the local latitude ( $\phi=32^{\circ} 46^{\prime} 49^{\prime \prime}$ for APO), $\delta$ is the target declination, $z$ is the zenith distance $\cos z=\sin \phi \sin \delta+\cos \phi \cos \delta \cos h$, and $\eta$ is defined in the range $-180^{\circ}$ to $+180^{\circ}$.

The SDSS $2.5 \mathrm{~m}$ telescope is equipped with an alt-az mount and all plates are observed with a position angle of $0^{\circ}$, so the amount of refraction in focal-plane coordinates ${ }^{28}$ is given by

$$
\begin{aligned}
& \Delta x_{\text {focal }}=-r \sin \eta \\
& \Delta y_{\text {focal }}=-r \cos \eta
\end{aligned}
$$

i.e., at transit $h=0, \eta=0^{\circ}$, and hence the entirety of the apparent refraction is along the $y$ focal direction. ${ }^{29}$

Since differential refraction (particularly shortward of $4000 \AA$ ) can be substantial compared to the fiber radius of 1 arcsec (see Figure 8) the spectrum recorded by a single fiber is not strictly the spectrum of a single region in a given galaxy; it is a bent "tube" that traces different regions of the galaxy at different wavelengths. Most immediately, this means that the effective on-sky footprint of the MaNGA IFUs can be shifted by up to $\sim 1$ arcsec between blue and red wavelengths, requiring that the MaNGA DRP (see D. R. Law et al. 2015, in preparation) rectify the spectra to a common astrometric grid when reconstructing the data cubes. More problematically, since the three exposures in a given dither set will be obtained

\footnotetext{
${ }^{28}$ SDSS $x$ focal/yfocal coordinates are defined such that $+x$ focal corresponds to + right ascension and $+y$ focal corresponds to +declination.

29 In the present work we neglect the relatively small effect of distortions introduced by the SDSS $2.5 \mathrm{~m}$ optical system; these are, however, accounted for in the actual data pipeline described by D. R. Law et al. (2015, in preparation).
} 
Coverage pattern: $5500 \AA$

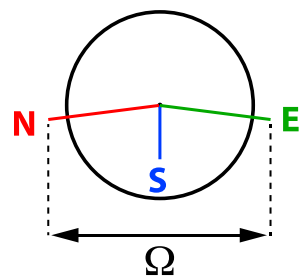

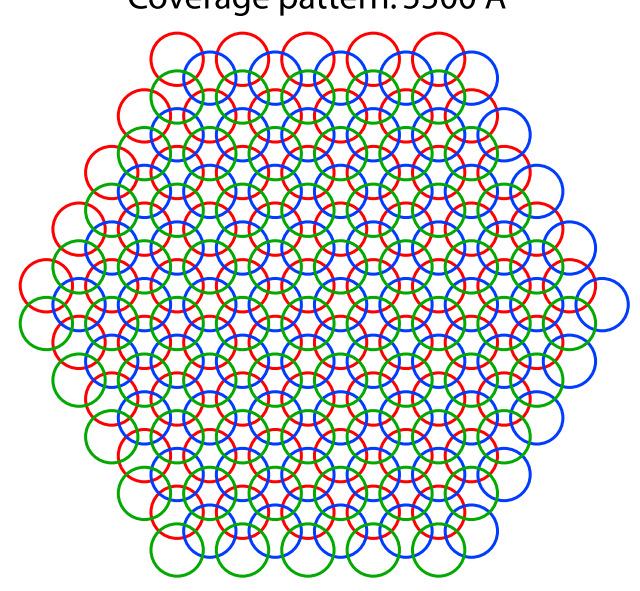

Coverage pattern: $3500 \AA$

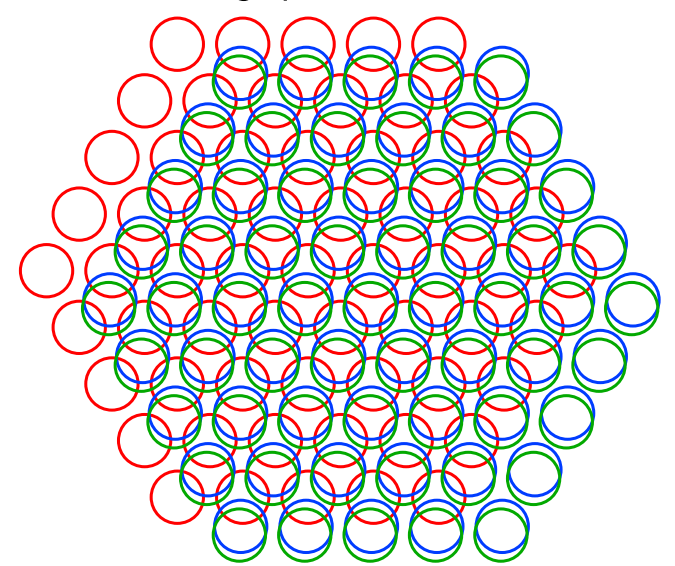

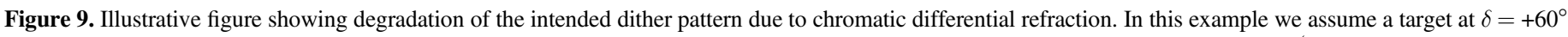

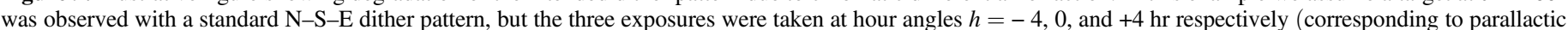

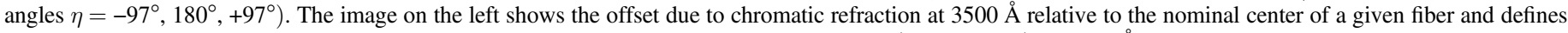

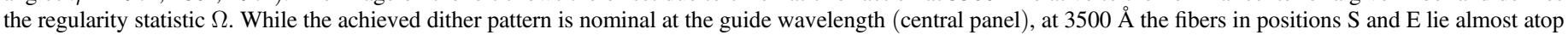
each other (right panel).

at different hour angles the relative offset at a given wavelength will change between these three exposures and degrade the intended dither pattern coverage.

At the guide wavelength of $5500 \AA$, the three dithers will be executed properly. As illustrated in Figure 9 however, at other wavelengths there will be variable shifts of the effective dithering pattern. These shifts can in some cases be comparable to the dither distances themselves, thereby degrading the effective dither pattern such that entire dither postions can be effectively "lost" at certain wavelengths. As suggested by Figures 5 and 7 this produces substantial and undesirable nonuniformities in the reconstructed image depth and recovered FWHM profile across the face of each IFU.

\subsection{Field Differential Refraction}

In addition to varying with wavelength, both the magnitude and the direction of atmospheric refraction vary according to the location of an object on the sky, and the $3^{\circ} \mathrm{SDSS}$ plugplate field over which our IFUs are distributed is sufficiently large that this variation cannot be neglected. As a given field rises, transits, and sets, the apparent locations of astronomical targets in the telescope focal plane shift. As described in Section 2, the SDSS telescope guider system compensates for this using guide fibers placed on astrometric standard stars distributed throughout a given field, and adjusts the overall shift, rotation, and scale of the focal plane to compensate. However, since field compression occurs along only a single direction (altitude) it cannot be fully corrected by a global change in the focal plane scale, leaving a residual quadrupole term in the guidercorrected focal plane locations of the target galaxies (see Figure 10). ${ }^{30}$

Such field differential effects are most noticeable when observing with single fibers or an array of slits (see, e.g., discussion by Cuby et al. 1998 , for the $16^{\prime} \times 16^{\prime}$ VIMOS field of view) since targets can rapidly shift out of the aperture. Hence, previous generations of SDSS that have used single fiber spectroscopy have been careful to observe at hour angles

\footnotetext{
30 Field differential refraction is calculated using the SDSS plate design code located on the collaboration SVN repository.
}

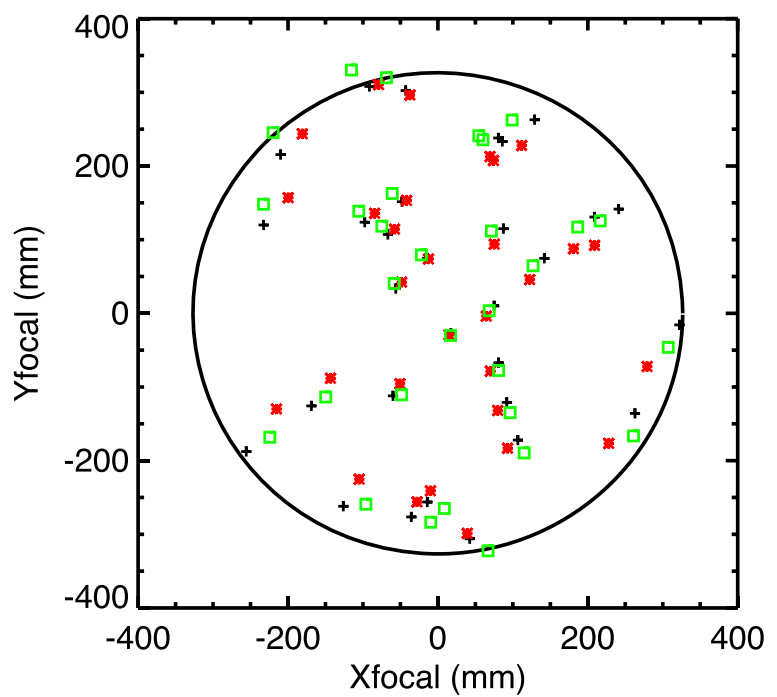

Figure 10. Magnified illustration of the effects of field differential refraction at the guide wavelength $(\sim 5500 \AA)$ across the $3^{\circ}$ diameter SDSS plugplate. Black "+" symbols indicate the nominal positions in focal plane coordinates of a randomly selected set of 30 target galaxies. These locations are computed assuming that the plate center has declination $+7^{\circ}$ and is observed at transit ( $h=0 \mathrm{hr}$ ); these correspond to the locations of the physically drilled holes in the plugplate into which the MaNGA IFUs are inserted. If the same plate were observed $4 \mathrm{hr}$ later $(h=+4 \mathrm{hr})$ the apparent locations of the galaxies in the focal plane would be different due to field differential refraction both before (red asterisks) and after (green open boxes) guider corrections have been applied. Note that all offsets from the nominal positions have been magnified by a factor of 300 to enhance visibility; the maximum actual shift after guider corrections in this example is $\sim 2$ arcsec.

close to that for which a given plate is drilled. In contrast, MaNGA is relatively insensitive to shifts in the effective centroid of an IFU since such shifts are small compared to the total field of view of each IFU ( 30 arcsec for the 127 fiber IFUs). ${ }^{31}$ The MaNGA plugplates are therefore all drilled for transit $(h=0 \mathrm{hr})$, so the holes into which the MaNGA IFUs are

\footnotetext{
31 This effect is more important for the spectrophotometric minibundles which have a diameter of only 7.5 arcsec; as discussed by R. Yan et al. (2015, in preparation), large offsets of the spectrophotometric standard stars from the center of the calibration minibundles due to a combination of differential refraction, dither offsets, and other effects can complicate flux calibration.
} 
inserted correspond to the expected focal plane locations of the galaxies at this point in time.

More important for MaNGA is the change in field differential refraction between exposures in a given dither set, which leads to degradation of the effective dither pattern akin to what was seen for chromatic differential refraction in Figure 9. As illustrated by Figure 10, the magnitude of this effect depends on the field declination, the hour angle $h$ of exposures within a given set, and the location of an IFU within the plugplate. In the extreme example shown in Figure 10 (low declination, with exposures obtained many hours apart) the shift can be comparable to a fiber diameter. In more realistic and typical cases (field center at $\delta=+40^{\circ}$, observed at $h=0$ and $h=+1 \mathrm{hr}$ ) the shift after guider corrections is typically $\lesssim 0.1$ arcsec.

\section{THE UNIFORMITY STATISTIC $\Omega$}

Given the presence of both chromatic and field differential refraction, no two exposures taken by MaNGA will have an identical fiber sampling pattern even in the absence of dithering. The primary driver of the MaNGA observing strategy is therefore mitigation of the impact of atmospheric differential refraction on the regularity of the dither pattern in order to achieve maximally uniform data quality and depth within a given IFU.

Given any two exposures separated by a time $\Delta t$ there are vectors $\boldsymbol{r}_{1}$ and $\boldsymbol{r}_{2}$ defining the effective offset of a fiber from its intended location on the target galaxy due to chromatic differential refraction, and $s_{1}$ and $s_{2}$ the offset due to uncorrectable field differential refraction effects. In our rectilinear focal-plane coordinate system the total shifts from differential refraction are given by:

$$
\begin{gathered}
\Delta x_{1}=r_{1} \sin \eta_{1}+\boldsymbol{x} \cdot \boldsymbol{s}_{1} \\
\Delta y_{1}=r_{1} \cos \eta_{1}+\boldsymbol{y} \cdot \boldsymbol{s}_{1} \\
\Delta x_{2}=r_{2} \sin \eta_{2}+\boldsymbol{x} \cdot \boldsymbol{s}_{2} \\
\Delta y_{2}=r_{2} \cos \eta_{2}+\boldsymbol{y} \cdot \boldsymbol{s}_{2}
\end{gathered}
$$

where $\eta_{1}$ and $\eta_{2}$ are the respective parallactic angles for the two exposures and the vectors $\boldsymbol{s}_{1}$ and $\boldsymbol{s}_{2}$ are each projected into their components along the $x / y$ focal plane coordinate system. The quantity of interest for survey planning purposes is the total distance between these shifted locations in the focal plane:

$$
\Omega=\sqrt{\left(\Delta x_{1}-\Delta x_{2}\right)^{2}+\left(\Delta y_{1}-\Delta y_{2}\right)^{2}} .
$$

In practice, we calculate $\Omega$ between the first and last exposures in a dithered set of three frames (see illustrative diagram in Figure 9). ${ }^{32}$ Using the tools developed in Section 3.3 we simulate four test cases where $\Omega$ ranges from 0 to $1^{\prime \prime}$. We use the as-built MaNGA 127 fiber IFU ma024, and assume a standard three-point $(\mathrm{N}-\mathrm{S}-\mathrm{E})$ dithering strategy in which exposure $\mathrm{N}$ is shifted by $\Omega / 2$ in the $-X$ focal direction, exposure $\mathrm{S}$ is shifted by $\Omega / 3$ in the $-Y$ focal direction and exposure $\mathrm{E}$ is shifted by $\Omega / 2$ in the $+X$ focal direction (see, e.g., Figure 9 ). Note that we are free to assume such symmetry because any shift common to all three exposures will simply result in a translation of the entire pattern.

\footnotetext{
32 Each exposure is 15 minutes in length; we adopt the midpoint of each exposure as the characteristic instant for purposes of calculating $\Omega$ (although see Section 7.2).
}

We show results for the expected exposure time, reconstructed PSF FWHM, and reconstructed axis ratio uniformity as a function of $\Omega$ in Figure $11 .{ }^{33}$ Figure 12 suggests that so long as $\Omega \lesssim 0.2$ arcsec observations should meet the target uniformity criteria outlined in Section 3.3.3 with FWHM $2.65 \pm 0.08$ arcsec. At $\Omega=0.4$ arcsec, degradations in the reconstructed PSF uniformity and circularity start to become apparent; although the mean reconstructed PSF in the bundle has FWHM $2.65 \pm 0.14$ arcsec the total spread of FWHM values can be as large as $\sim 0.3$ arcsec, and $99 \%$ of locations have minor/major axis ratio greater than 0.85 . By $\Omega=1.0$ arcsec the dither pattern is badly degraded, with reconstructed FWHM values varying by over an arcsecond depending on where a point source falls within the bundle. Our science requirements (Section 3.1) therefore translate to a requirement that $\Omega<0.4$ arcsec, with the goal of reaching $\Omega<0.2$ arcsec for the majority of observations so that it does not dominate the flux calibration accuracy budget.

\section{MaNGA OBSERVING STRATEGY}

\subsection{Set Lengths and Visibility Windows}

As described above, $\Omega$ is a complicated function of wavelength, integration time, target declination, hour angle, and location of an IFU on a given plate. However, it is possible to define a series of relatively simple observing guidelines that will ensure that $\Omega$ stays below our 0.4 arcsec threshold.

First, we note that $\Omega$ behaves nearly linearly with the amount of elapsed time between exposures in a given set, meaning that it is desirable to obtain all three exposures in the set as close in time to each other as possible. Since each exposure is 15 minutes long, we therefore require that all three exposures be obtained in a set length of $1 \mathrm{hr}$ (i.e., the change in hour angle between the start of the first exposure and the end of the last exposure should be $1 \mathrm{hr}$ or less, corresponding to 45 minutes between the effective midpoint of the first and last exposure). While we expect that each set of three exposures will typically last 48 minutes accounting for typical readout times and overheads, this hour-long block provides necessary flexibility in scheduling, especially during variable weather conditions.

We next calculate the expected $\Omega$ within a $1 \mathrm{hr}$ long set as a function of the midpoint hour angle $h_{\text {set }}$ of the set $\left(h_{\text {set }}\right.$ denotes the absolute value of the hour angle midway between the start of the first and end of the last exposure). In Figure 12 we show the results of this calculation for three different wavelengths, three locations on a plate, and a range of different declinations. ${ }^{34}$ As expected, $\Omega$ is largest at extremely blue wavelengths (for which chromatic differential refraction is greatest) and on the edges of a plate (where uncorrected field differential refraction is greatest). More importantly however, we note that $\Omega$ grows rapidly with increasing hour angle (either east or west of the meridian) meaning that we want to obtain our observations as close to transit as possible. Our $\Omega$ limit

\footnotetext{
33 Note that while $\Omega$ degrades the expect coverage pattern, we assume that the magnitude and direction of all of these shifts are known (see discussion by D. R. Law et al. 2015, in preparation) and the true effective locations of each fiber are used when reconstructing the data cube.

34 Due to symmetries inherent in this exercise (chromatic and field differential effects combining constructively or destructively), at fixed wavelength one side of the plate will exhibit the worst $\Omega$ at positive hour angles (west of meridian) and the other at negative hour angles (east of meridian). For convenience we collapse the problem such that $h_{\text {set }}$ refers to the absolute value of the hour angle, and $\Omega$ is taken to be the greater of the value from $\pm h_{\text {set }}$.
} 


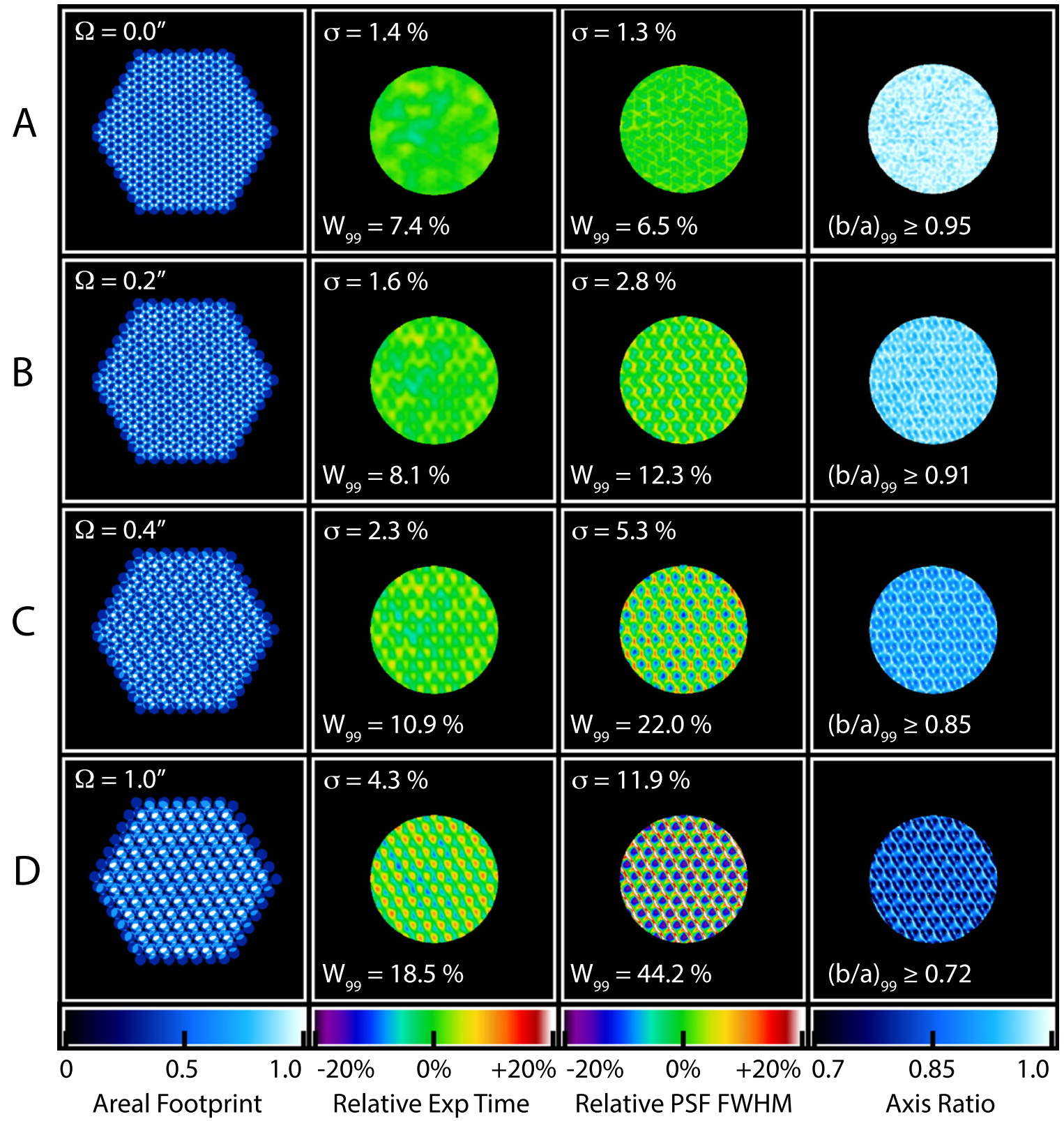

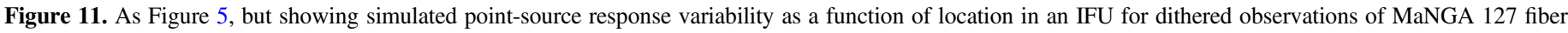
bundle ma024 with different values of the pattern degradation $\Omega$.

therefore equates to defining a series of visibility windows around transit within which all MaNGA observations must be taken.

In order to compute the length of these visibility windows we require that $\Omega$ must be less than 0.4 arcsec for all sets, at all wavelengths, at all locations on a given plate, and at all declinations. As indicated by Figure 12, the worst wavelength for $\Omega$ will be $3600 \AA$, where the chromatic refraction is greatest. We work out the worst location on a given plate as a function of declination by using Monte Carlo techniques to compute $\Omega$ for each of 20,000 randomly chosen locations on an SDSS plugplate over the course of a $1 \mathrm{hr}$ set. As illustrated by Figure 13, the worst $\Omega$ is typically for IFUs located on the eastern/western edges of the plate for target declinations $\sim$ $+30^{\circ}-40^{\circ}$; this pattern shifts at more northerly/southerly declinations.
Using these simulations we finally have all of the pieces required to define our visibility windows. For a grid of declinations spaced every $5^{\circ}$ from $\delta=0^{\circ}$ to $70^{\circ}$ we compute the limiting set hour angle such that $\Omega=0.4$ arcsec at $\lambda=3600 \AA$ at the worst location on a given plate. Converting the set midpoint hour angle to the maximum midpoint hour angle of an individual exposure $\left(h_{\text {exp }}=h_{\text {set }}+22.5\right.$ minutes for $1 \mathrm{hr}$ sets), we show the final visibility windows as a function of declination in Figure 14. These windows range from about $1 \mathrm{hr}$ either side of transit for fields near the celestial equator to $\sim 3 \mathrm{hr}$ for declinations $\delta \sim+40^{\circ}$.

Intriguingly, despite all of the complications involved in computing these visibility windows they are nearly equivalent to simple airmass limits, independent of field declination. As illustrated by Figure 14, our visibility windows can be described as a 6th order polynomial as a function of declination, or more 


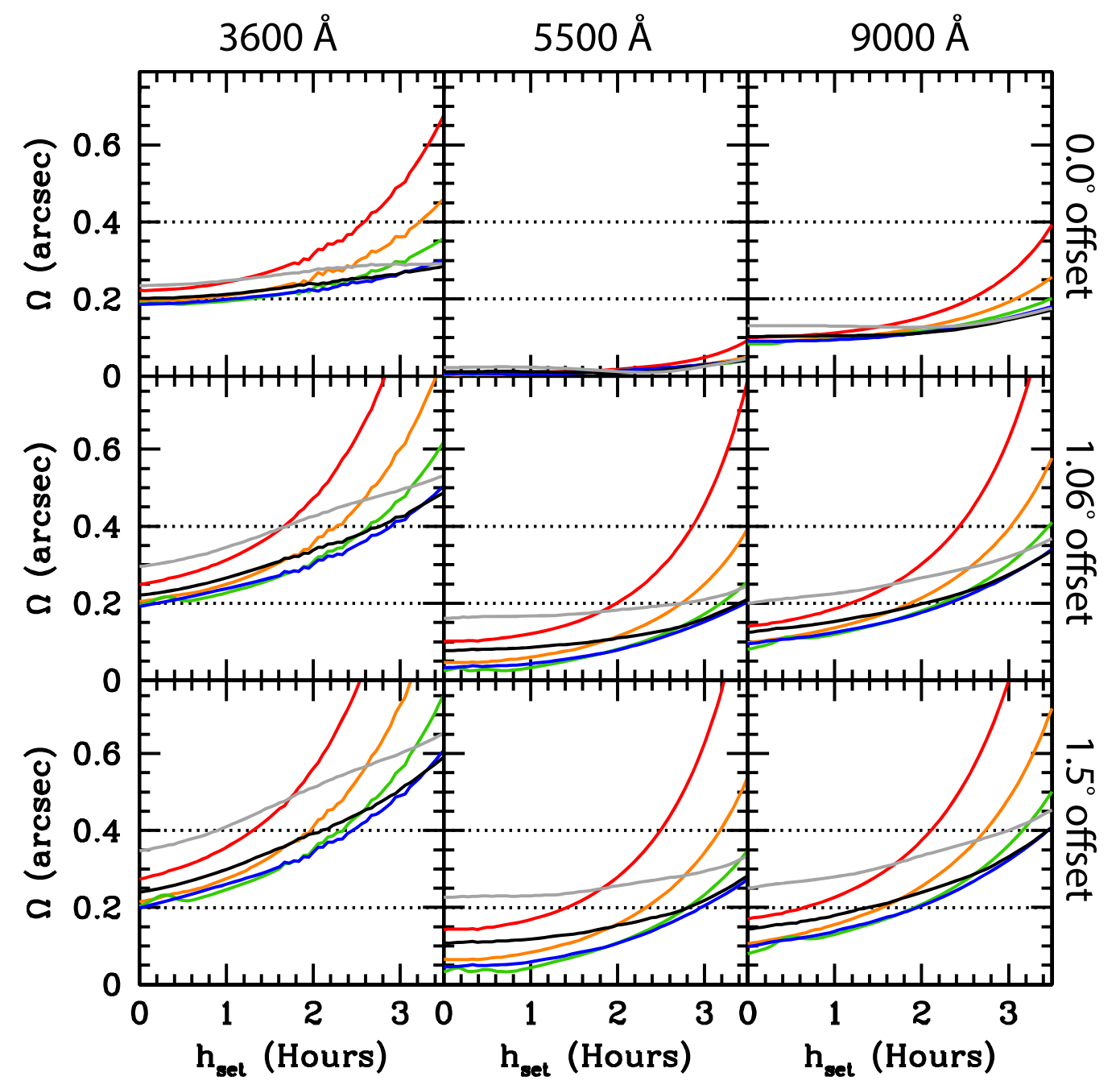

Figure 12. $\Omega$ statistic as a function of midpoint hour angle of the set $\left(h_{\text {set }}\right)$ for a range of wavelengths, target declinations, and locations on a plate. Left, middle, and right columns respectively show results for wavelengths of 3600,5500 , and $9000 \AA$; top, middle, and bottom row respectively show results for an IFU in the middle of the plate, 1:06 toward the E edge of the plate (a circle at this radius encloses 50\% of the plate area), and on the E edge of the plate. Red, orange, green, blue, black, and gray solid lines respectively indicate results for declinations $\delta=0^{\circ}, 15^{\circ}, 30^{\circ}, 45^{\circ}, 60^{\circ}$, and $75^{\circ}$. The horizontal dotted lines at $\Omega=0.2$ and 0.4 indicate the thresholds of ideal and acceptable performance respectively. High-frequency structure in some lines is due to discrete changes in the best-fit guider corrections between individual simulation points.

simply by the requirement that airmass $\mathrm{AM}<1.21$ for all exposures at all declinations. This airmass limit is determined by the SDSS plate diameter, the BOSS spectrograph wavelength coverage, and the assumed length of each set. ${ }^{35}$

We note that while these visibility windows have been established to ensure that $\Omega<0.4$ arcsec at all wavelengths for all MaNGA observations, typical performance is expected to be considerably better than this. At most wavelengths, most locations on a plate, and most hour angles within the visibility window $\Omega$ will be 0.2 arcsec or below (see, e.g., Figures 12 and 13). Additionally, these simulations have assumed that sets are completed in one hour ( 45 minutes between the midpoint of first and last exposures in a set). Early survey observations at

\footnotetext{
35 It is therefore possible to increase the airmass limit by reducing the set length or effective plate diameter (i.e., restricting the locations of IFUs on the plate). For instance, a set length of 48 minutes instead of $1 \mathrm{hr}$ would increase the airmass limit to 1.34 , expanding the visibility windows significantly. Such modifications to the observing strategy set forth here will be actively explored over the lifetime of the survey.
}

APO suggest efficiency such that most sets are actually observed in more like 48 minutes ( 33 minutes between the midpoint of first and last exposures); since $\Omega$ scales roughly linearly with the set length we therefore expect on-sky performance to typically be a factor $\sim 33 \%$ better than assumed in these simulations. Additionally, irregular coverage of an astronomical target in one set of exposures will tend to be averaged out across many such sets, resulting in more uniform performance for the final data cube of a given source.

\subsection{Observing Conditions and Missing Exposures}

Thus far, all simulations have assumed that atmospheric seeing remains constant throughout all exposures in a given set, and that small variations in transparency can be normalized via per-exposure flux calibration (although see Section 7.4). This assumption is often reasonable over the course of any given hour, but since rapid changes in observing conditions occur on 


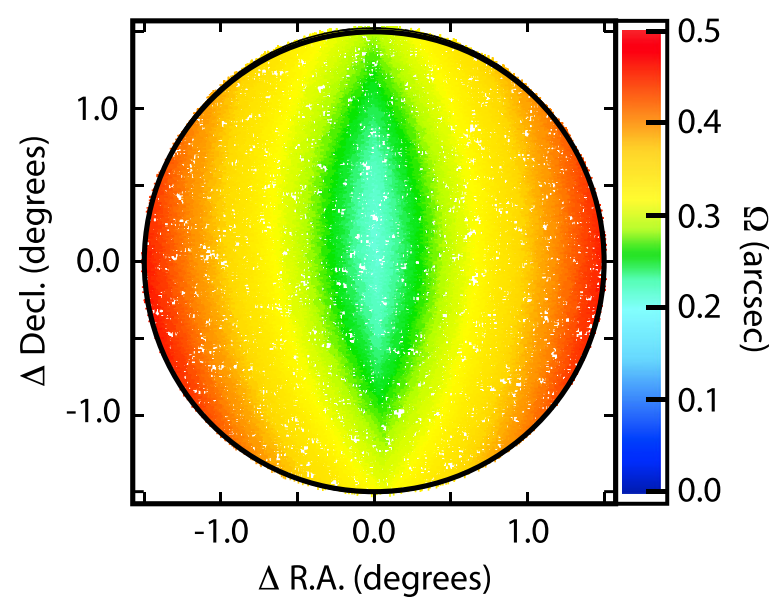

Figure 13. $\Omega$ as a function of location on a plate centered at $\delta=40^{\circ}$. Simulations are performed at $3600 \AA$ and assume a $5 \mathrm{hr}$ observing window (i.e., $h_{\text {set }}=2.5 \mathrm{hr}$ either side of transit). Each point represents the maximum value of $\Omega$ experienced at a given location for a hour-long set of exposures taken within this observing window (for one side of the plate this maximum will occur prior to transit, for the other side it will occur after transit).

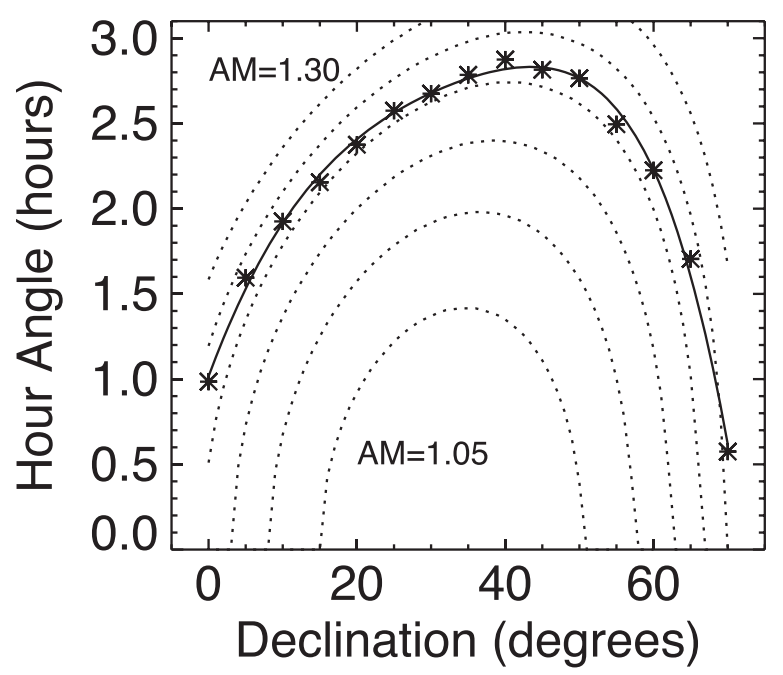

Figure 14. Black asterisks show the maximum hour angle away from transit $\left(h_{\text {exp }}\right)$ within which all MaNGA exposures must be obtained as a function of declination based on numerical simulations. The solid black line represents a polynomial fit to these 15 data points. Dotted lines indicate contours of constant airmass (every 0.05 from AM 1.05 to 1.30 ) as a function of declination and hour angle; note that these contours closely track the derived hour angle limits.

some nights we must formulate our observing strategy accordingly.

Consider, for instance, the pathological case where two dithered exposures have been successfully obtained in good conditions, but the third is lost. Whether it is never taken, or taken in extremely poor conditions (e.g., heavy cloud, seeing greater than 4 arcsec FWHM, etc.), the combined set of exposures no longer uniformly samples the source image. In such a situation, the missing exposure would have to be made up on another night, and obtained within a small range of allowable hour angles such that the total set length is still less than one hour.

We therefore establish a series of additional requirements for image uniformity across exposures within a given set. Based on simulations similar to those described in Sections 3.3 and 5 but allowing for variable seeing and transparency, we find the following.

1. All exposures in a set should have seeing within 0.8 arcsec of each other.

2. All exposures in a set should have $(\mathrm{S} / \mathrm{N})^{2}$ values within a factor of 2 of each other.

3. Each set of exposures should have median seeing 2.0 arcsec or below in order for the reconstructed image to have FWHM less than 3 arcsec (ensuring uniformity of image quality between galaxies in the MaNGA survey).

Historical conditions at APO and experience during MaNGA commissioning suggest that atmospheric conditions are generally stable enough that these criteria will not pose a serious limitation to survey operations. In practice, exposures also can often be rearranged between sets to optimize observing efficiency and minimize the need for patching of missing dither positions (see discussion by R. Yan et al. 2015, in preparation), and further modifications to the baseline strategy will continue to be explored throughout the survey.

\section{ADDITIONAL CONSIDERATIONS}

Although differential refraction considerations are the primary factor that sets the MaNGA observing strategy, we also highlight a few additional considerations here that will impact the MaNGA reconstructed image quality and must be accounted for in survey operations.

\subsection{Required Dithering Accuracy}

Just as differential refraction effects degrade the effective dithering pattern and contribute to non-uniform sampling of an astronomical source, so too does the dithering accuracy of the telescope. As described above in Section 6.1, $\Omega$ from refractive sources will frequently be less than $0.1-0.2$ arcsec, and the individual telescope offsets must therefore be good to better than 0.1 arcsec in order to not be the limiting factor governing the image sampling regularity for the majority of observations. Indeed, it is particularly important to minimize the contribution of offsetting errors for cases with already-high $\Omega$ from differential refraction as the compounded errors may easily make the difference between an acceptably versus unacceptably uniform set of exposures. Based on observations performed at APO during MaNGA commissioning, ${ }^{36}$ the dither offset error has a median of $0.063 \mathrm{arcsec}$, and is smaller than 0.1 arcsec in $76 \%$ of exposures. Although the current dithering accuracy degrades to a median of $0.1 \mathrm{arcsec}$ at altitudes higher than $80^{\circ}$, work is ongoing to improve this performance (see details in R. Yan et al. 2015, in preparation).

\subsection{Required Guiding Accuracy}

In addition to the accuracy with which the telescope offsets are performed it is also important to consider the guiding accuracy of the telescope (i.e., how well a given position is maintained over the course of an exposure). Although poor guiding performance will not degrade the coverage uniformity of a set of exposures, it will degrade the image quality of the

\footnotetext{
36 The guider system uses 16 coherent imaging fiber bundles plugged on the plate and imaged by a separate guider camera; by monitoring the positions of these 16 stars and comparing them to the desired positions we can measure the dithering accuracy directly.
} 


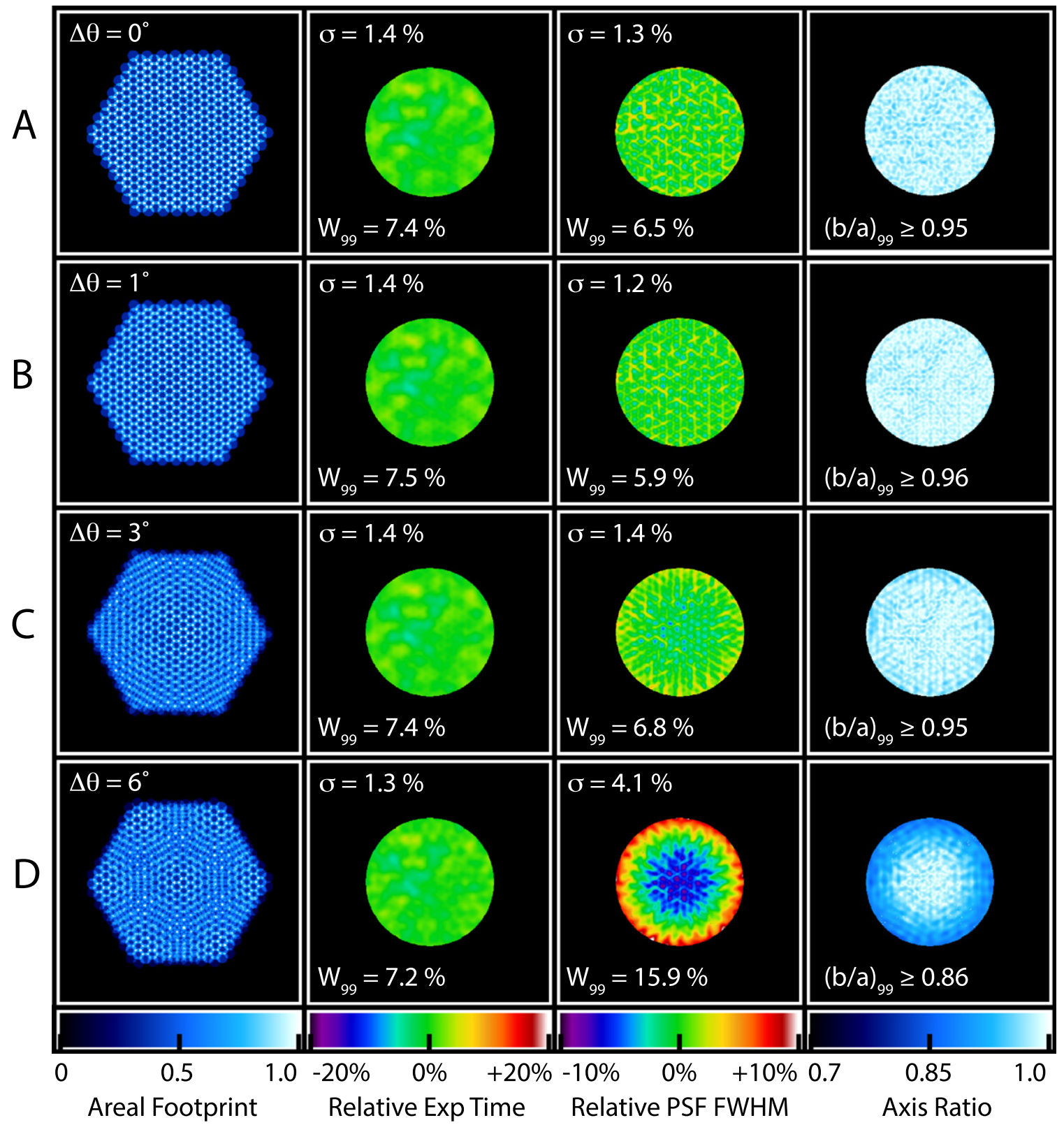

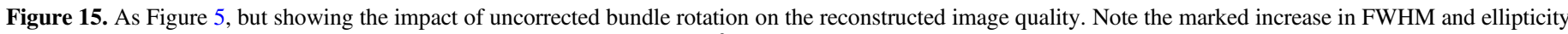
of reconstructed point sources near the edges of the bundle for offsets $\sim 6^{\circ}$.

exposures by contributing in quadrature to the effective astronomical seeing. Observations obtained during MaNGA commissioning show that the median guiding accuracy (based on variations in guide star positions across all $15 \mathrm{~s}$ guider camera exposures during each 15 minute science exposure; see details in R. Yan et al. 2015, in preparation) is 0.12 arcsec, substantially smaller than the median SDSS $2.5 \mathrm{~m}$ seeing of $\sim 1^{\prime \prime} .5$ (computed across all BOSS spectroscopy in 2012).

We note that a similar effect is caused by differential refraction; just as changing refraction causes the effective location of a fiber to move between two exposures (Section 4.2), so too does it cause the effective location of a fiber on a given astronomical target to move during the exposure as well. However, for observations obtained using the strategy outlined in Section 6 above this effect is small. Since differential motion of a fiber with respect to a fiducial position (i.e., $\Omega$ ) scales roughly linearly with time, the motion in a
15 minute exposure will be $\sim 1 / 3$ of that for a given set of 3 exposures. Since we require the latter to be $<0.4$ arcsec even in extreme cases, the motion in a single exposure will be $\lesssim 0.1$ arcsec, which is small compared to the guider accuracy and atmospheric seeing profile.

\subsection{Bundle Rotation}

The rotational position $\theta$ of the MaNGA IFU bundles is controlled via clocking pins that ensure proper alignment of each IFU. However, mechanical tolerances of the pinhole translate to a rotational uncertainty for each bundle at the level of $\sim 3^{\circ}$. In order to ensure that individual sets meet the $\Omega<0.2$ arcsec coverage regularity goal at the edges of the largest fiber bundles ( $\sim 16.5$ arcsec radius) we specify that the rotational offset $\Delta \theta$ required between any two exposures in a 
given set be

$$
\Delta \theta<\tan ^{-1}(0.2 / 16.5)=0.7 .
$$

Generally, rotational tension in the IFU cables should ensure that $\theta$ remains relatively constant for a given plugging, and preliminary tests indicate that $\Delta \theta \approx 0: 2$ (see $\mathrm{D}$. R. Law et al. 2015 , in preparation for further details). However, changes in the routing path of each IFU cable through the cartridge can lead to a different rotation (and small translational offsets) each time the plate is replugged, and we therefore require that sets be completed within a single plugging.

Rotation between sets of exposures can also degrade the reconstructed image quality if it is not measured and accounted for in the final astrometric solution. In Figure 15 we simulate the effect of stacking together two sets with different rotations without accounting for their rotational offset in the fiber astrometry. Visible degradation of the reconstructed PSF starts to become apparent at the edges of the IFU bundle once the sets are rotated from each other by $\sim 3^{\circ}$, and distortions become severe once the rotation reaches $6^{\circ}$ (i.e., $\sim$ a fiber radius at the edge of the largest bundles). We therefore require that the MaNGA data pipeline be able to measure the rotational clocking of each IFU at the level of $\sim \pm 1^{\circ}$ so that it can be incorporated into the astrometric solution.

\subsection{Errors in Spectrophotometry}

As discussed by D. R. Law et al. (2015, in preparation) and R. Yan et al. (2015, in preparation), each MaNGA exposure is flux calibrated independently to account for variations in the atmospheric seeing and transparency. Adequate image reconstruction is therefore dependent on the relative accuracy of the flux calibration between exposures in a given set; any offsets between exposures will hamper the ability of the dithered exposures to properly sample the source profile. The most pronounced effect of such offsets is not their degradation of the spatial profile of unresolved structures (e.g., point sources) however, but rather their introduction of artificial spatial structure into a smooth background.

We therefore simulate dithered observations of a constant surface-brightness source (e.g., like the outskirts of a smooth elliptical galaxy), assuming typical observing conditions with visual seeing $\sim 1.5$ arcsec. We mimic flux calibration errors by multiplying the fiber fluxes for each exposure by a scale factor drawn randomly from a gaussian distribution with a given rms width and a median of 1.0 before reconstructing the composite image. As illustrated in Figure 16, calibration errors between individual exposures results in a stippling of the smooth background, introducing artificial spatial structure correlated with the dithered fiber pattern.

In a single set of 3 exposures, we find that rms flux calibration errors of $2 \%$ between exposures results in a reconstructed image whose surface brightness varies by $0.4 \%$ (rms) from pixel to pixel. This is comparable to the $0.3 \%$ pixelto-pixel variations that we find in the reconstructed image assuming perfect flux calibration of all exposures. As flux calibration accuracy degrades further to 5\%,15\%, and 50\% rms between exposures, we find pixel-to-pixel variations of $1 \%$, $2 \%$, and $8 \%$ respectively in the reconstructed image. If flux calibration errors are uncorrelated between exposures in different sets, this variation will average out over the course of observations for a given plate. Even in the case where individual exposures are calibrated as poorly as to within a

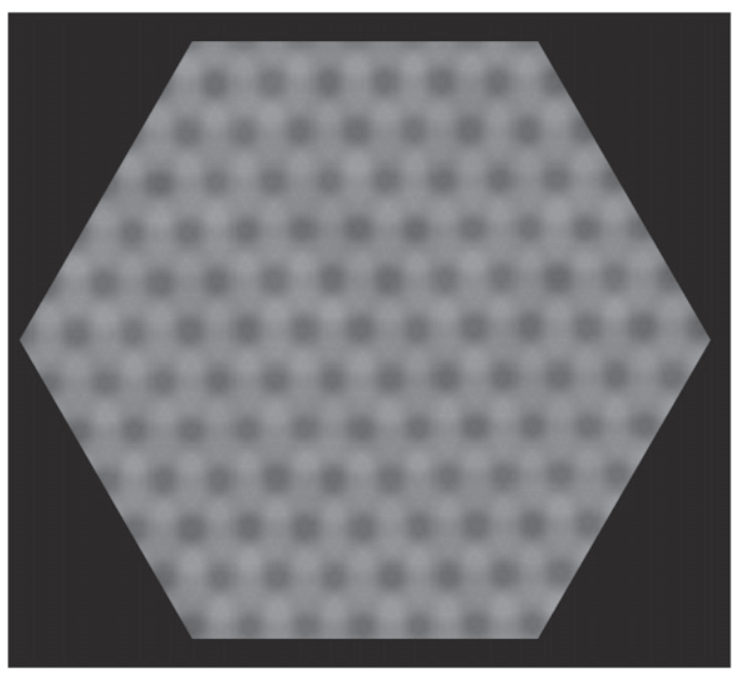

Figure 16. Simulated MaNGA observation of a constant surface-brightness field showing the characteristic stippling pattern introduced by relative flux calibration errors between dithered exposures. Such errors introduce artificial spatial structure correlated with the dithered fiber pattern. Grayscale stretch is arbitrary; in this example the blackpoint (whitepoint) is set to $20 \%$ below (above) the mean flux, corresponding to an rms variation of about $8 \%$ over the field of view. Note that the simulated field has been trimmed to omit effects from regions at the edge of the IFU field.

factor of 2, the pixel-to-pixel flux for a uniform background source varies by just $2 \% \mathrm{rms}$ when averaged over 4 sets (12 total exposures). In contrast, preliminary results from MaNGA commissioning data indicate that individual exposures are typically calibrated to within $2.5 \%$ (R. Yan et al. 2015, in preparation), suggesting that flux calibration errors are unlikely to contribute significantly to the image reconstruction fidelity.

\section{SUMMARY}

The MaNGA hardware design and observing strategy is driven by the desire to ensure high, uniform image quality and depth across all 10,000 of the galaxies that will be observed during SDSS-IV. In particular, the goal of reaching $7 \%$ spectrophotometric accuracy between $\left[\mathrm{O}_{\mathrm{II}}\right] \lambda 3727$ and $\mathrm{H} \alpha \lambda$ 6564 requires that the reconstructed PSF varies by $10 \%$ or less (in both width and ellipticity) across the face of each IFU. This goal is particularly challenging given the variable total number of exposures per field $(\sim 6-20)$ required to reach the target depth, chromatic differential refraction arising from the large wavelength coverage of the survey $(\lambda \lambda 3600-10000 \AA)$, and field differential refraction caused by the $3^{\circ}$ wide field of view over which individual IFUs are deployed.

We summarize the requirements necessary to meet our goal as follows.

1. Each IFU fiber bundle should be constructed of a regular hexagonal grid of fibers to an accuracy of $5 \mu \mathrm{m} \mathrm{rms}$. The MaNGA IFUs meet and exceed this specification with a filling factor of $56 \%$ and a typical fiber placement accuracy of $\sim 3 \mu \mathrm{m} \mathrm{rms}$.

2. Exposures should be obtained in sets of three 15 minute exposures dithered to the vertices of an $1^{\prime \prime}$.44 equilateral triangle in order for each set to uniformly sample the image plane.

3. The telescope must be able to dither to an accuracy of $0^{\prime \prime} .1$ or better. 
4. Each plate should be observed for an integer number of sets until the combined depth reaches a S/N of $5 \AA^{-1}$ fiber ${ }^{-1}$ in the $r$-band continuum at a surface brightness of $23 \mathrm{AB} \operatorname{arcsec}^{-2}$.

5. All three exposures in a set must be observed within one hour of each other (i.e., the change in hour angle between the start of the first exposure and the end of the last exposure should be $1 \mathrm{hr}$ or less), and in a single plugging of a given plate.

6. All three exposures in a set should have $(\mathrm{S} / \mathrm{N})^{2}$ within a factor of 2 of each other, and be obtained in atmospheric seeing that varies by less than 0 ". 8 . Each set should be obtained in median seeing of 2 ". 0 or better.

7. All MaNGA exposures should be obtained in visibility windows $\sim 2-6 \mathrm{hr}$ in length corresponding to airmass $\leqslant 1.21$.

8. MaNGA relative flux calibration between exposures must be good to $\sim 5 \%$ or better.

In reality, many of the issues considered here will tend to average out over the course of the 3-4 sets that will typically be obtained on a given plate since effects that cause a slight gap in coverage for one set will often be filled in by another. However, our objective in designing the MaNGA program is to ensure that the depth, coverage, and image quality of the entire survey is as good and uniform as possible for the entire wavelength range of each of our 10,000 galaxies. In forthcoming contributions (D. R. Law et al. 2015, in preparation, R. Yan et al. 2015, in preparation) we will explore in greater detail how well we succeed in meeting these goals.

We thank Maryna Tsybulska for early contributions to this project, and acknowledge the Summer Undergraduate Research Program at the Dunlap Institute, University of Toronto for their support. A.W. acknowledges support of a Leverhulme Trust Early Career Fellowship. D.R.L. thanks the anonymous referee whose comments led to an improved version of the manuscript. Funding for the Sloan Digital Sky Survey IV has been provided by the Alfred P. Sloan Foundation and the Participating Institutions. SDSS-IV acknowledges support and resources from the Center for High-Performance Computing at the University of Utah. The SDSS web site is www.sdss.org. SDSS-IV is managed by the Astrophysical Research Consortium for the Participating Institutions of the SDSS Collaboration including the Carnegie Institution for Science, Carnegie Mellon University, the Chilean Participation Group,
Harvard-Smithsonian Center for Astrophysics, Instituto de Astrofísica de Canarias, The Johns Hopkins University, Kavli Institute for the Physics and Mathematics of the Universe (IPMU)/University of Tokyo, Lawrence Berkeley National Laboratory, Leibniz Institut für Astrophysik Potsdam (AIP), Max-Planck-Institut für Astrophysik (MPA Garching), MaxPlanck-Institut für Extraterrestrische Physik (MPE), MaxPlanck-Institut für Astronomie (MPIA Heidelberg), National Astronomical Observatory of China, New Mexico State University, New York University, The Ohio State University, Pennsylvania State University, Shanghai Astronomical Observatory, United Kingdom Participation Group, Universidad Nacional Autónoma de México, University of Arizona, University of Colorado Boulder, University of Portsmouth, University of Utah, University of Washington, University of Wisconsin, Vanderbilt University, and Yale University.

\section{REFERENCES}

Bacon, R., Copin, Y., Monnet, G., et al. 2001, MNRAS, 326, 23 Bacon, R., Accardo, M., Adjali, L., et al. 2010, Proc. SPIE, 7735, 773508

Baldwin, J. A., Phillips, M. M., \& Terlevich, R. 1981, PASP, 93, 5

Belfiore, F., Maiolino, R., Bundy, K., et al. 2015, MNRAS, 449, 867

Bershady, M. A., Verheijen, M. A. W., Westfall, K. B., et al. 2010, ApJ, 716,234

Bland-Hawthorn, J., Bryant, J., Robertson, G., et al. 2010, Proc. SPIE, 7735, 773541

Bolton, A. S., Schlegel, D. J., Aubourg, É, et al. 2012, AJ, 144, 144

Bryant, J. J., Bland-Hawthorn, J., Fogarty, L. M. R., Lawrence, J. S., \& Croom, S. M. 2014, MNRAS, 438, 869

Bundy, K., Bershady, M. A., Law, D. R., et al. 2015, ApJ, 798, 7

Croom, S. M., Lawrence, J. S., Bland-Hawthorn, J., et al. 2012, MNRAS, 421,872

Cuby, J. G., Bottini, D., \& Picat, J. P. 1998, Proc. SPIE, 3355, 36

Dawson, K. S., Schlegel, D. J., Ahn, C. P., et al. 2013, AJ, 145, 10

Drory, N., MacDonald, N., Bershady, M. A., et al. 2015, AJ, 149, 77

Emsellem, E., Cappellari, M., \& Peletier, R. F. 2004, MNRAS, 352, 721

Eisenhauer, F., Abuter, R., Bickert, K., et al. 2003, Proc. SPIE, 4841, 1548

Fabricius, M. H., Coccato, L., Bender, R., et al. 2014, MNRAS, 441, 2212

Filippenko, A. V. 1982, PASP, 94, 715

Gunn, J. E., Siegmund, W. A., Mannery, E. J., et al. 2006, AJ, 131, 2332

Larkin, J. E., Quirrenbach, A., Krabbe, A., et al. 2003, Proc. SPIE, 4841, 1600

Law, D. R., Steidel, C. C., Erb, D. K., et al. 2009, ApJ, 697, 421

Li, C., Wang, E., Lin, L., et al. 2015, ApJ, 804, 125

Sánchez, S. F., Kennicutt, R. C., Gil de Paz, A., et al. 2012, A\&A, 538, AA8

Sharp, R., Allen, J. T., Fogarty, L. M. R., et al. 2015, MNRAS, 446, 1551

Sharples, R., Bender, R., Berbel, A., et al. 2013, Msngr, 151, 21

Smee, S. A., Gunn, J. E., Uomoto, A., et al. 2013, AJ, 146, 32

Weijmans, A.-M., de Zeeuw, P. T., Emsellem, E., et al. 2014, MNRAS, 444, 3340

Wilkinson, D. M., Maraston, C., Thomas, D., et al. 2015, MNRAS, 449, 328 